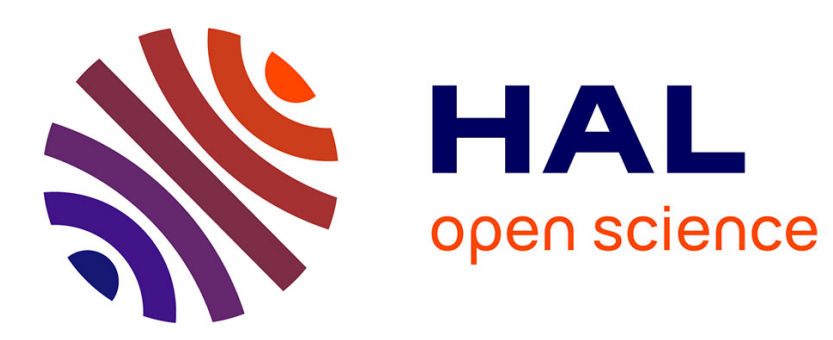

\title{
Price Dynamics in Market with Heterogeneous Investment Horizons and Boundedly Rational Traders
}

Thierry Chauveau, Alexander Subbotin

\section{To cite this version:}

Thierry Chauveau, Alexander Subbotin. Price Dynamics in Market with Heterogeneous Investment Horizons and Boundedly Rational Traders. 2010. halshs-00497427

\section{HAL Id: halshs-00497427 https://shs.hal.science/halshs-00497427}

Submitted on 5 Jul 2010

HAL is a multi-disciplinary open access archive for the deposit and dissemination of scientific research documents, whether they are published or not. The documents may come from teaching and research institutions in France or abroad, or from public or private research centers.
L'archive ouverte pluridisciplinaire HAL, est destinée au dépôt et à la diffusion de documents scientifiques de niveau recherche, publiés ou non, émanant des établissements d'enseignement et de recherche français ou étrangers, des laboratoires publics ou privés. 


\section{Documents de Travail du Centre d'Economie de la Sorbonne}
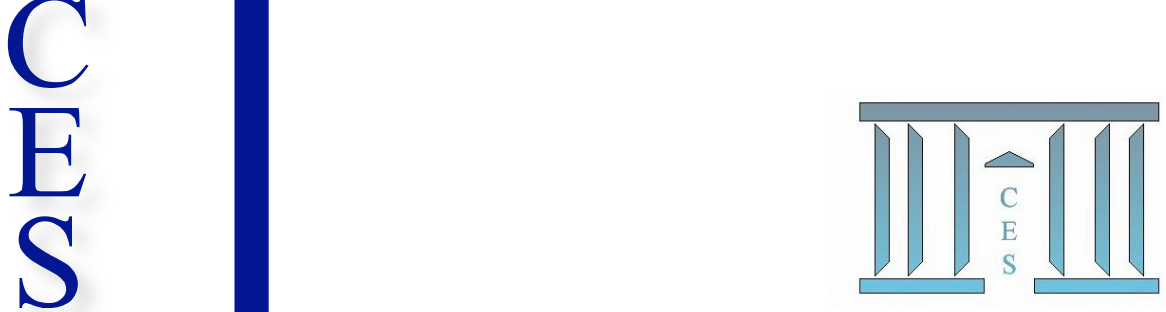

Price Dynamics in a Market with Heterogeneous Investment Horizons and Boundedly Rational Traders

Alexander SubBotin, Thierry CHAUVEAU

2010.48

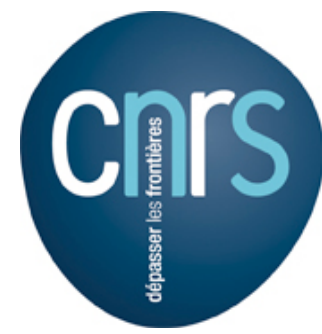

Maison des Sciences Économiques, 106-112 boulevard de L'Hôpital, 75647 Paris Cedex 13 


\title{
Price Dynamics in a Market with Heterogeneous Investment Horizons and Boundedly Rational Traders *
}

\author{
Alexander Subbotin ${ }^{\dagger} \quad$ Thierry Chauveau ${ }^{\ddagger}$
}

April 10, 2010

\begin{abstract}
This paper studies the effects of multiple investment horizons and investors' bounded rationality on the price dynamics. We consider a pure exchange economy with one risky asset, populated with agents maximizing CRRA-type expected utility of wealth over discrete investment periods. An investor's demand for the risky asset may depend on the historical returns, so that our model encompasses a wide range of behaviorist patterns. The necessary conditions, under which the risky return can be a stationary iid process, are established. The compatibility of these conditions with different types of demand functions in the heterogeneous agents' framework are explored. We find that conditional volatility of returns cannot be constant in many generic situations, especially if agents with different investment horizons operate on the market. In the latter case the return process can display conditional heteroscedasticity, even if all investors are so-called "fundamentalists" and their demand for the risky asset is subject to exogenous iid shocks. We show that the heterogeneity of investment horizons can be a possible explanation of different stylized patterns in stock returns, in particular, mean-reversion and volatility clustering.
\end{abstract}

Keywords: Asset Pricing, Heterogeneous Agents, Multiple Investment Scales, Volatility Clustering

J.E.L. Classification: G.10, G.14.

\section{Résumé}

Nous étudions l'effet de multiples horizons d'investissement et de la rationalité limitée des investisseurs sur la dynamique des prix. Dans le cadre d'un modèle à agents hétérogènes, nous introduisons de multiples échelles d'investissement. Nous trouvons que la volatilité conditionnelle des rendements ne peut pas être constante dans de nombreuses situations génériques, en particulier si les agents avec des horizons d'investissement différents opèrent sur le marché. Dans ce dernier cas, le processus de rendements peut être hétéroscédastique, même si tous les investisseurs sont "fondamentalistes" mais leur demande de l'actif risqué est soumise à des chocs iid exogènes. Nous montrons que l'hétérogénéité des horizons d'investissement peut être une explication possible de différents faits stylisés, observés pour les rendements des actions et, en particulier, du retour à la moyenne et du clustering de la volatilité.

Mots clés: Evaluation des actifs, agents hétérogènes, multiples horizons d'investissement, clustering de volatilité.

\section{J.E.L. Classification: G10, C13.}

*The authors thank K. Shapovalova, M. Anufirev, C.Hommes, R.Topol, Y. Mirkin and B. de Meyer for helpful remarks.

†University of Paris-1 (CES/CNRS), e-mail: alexander.subbotin@univ-paris1.fr

$\ddagger$ University of Paris-1 (CES/CNRS), e-mail: thierry.chaveau@univ-paris1.fr 


\section{Introduction}

Up to now, the heterogeneous markets literature almost exclusively focuses on the expectations of market agents, according to which investors are classified into "fundamentalists", "chartists" and "noise traders". It is shown that the interaction, herding behavior and strategy switching of heterogeneous agents transform noise process and create persistent trading volume, excess volatility, fat tails, clustered volatility, scaling laws (see Hommes [2006] and LeBaron [2006] for surveys on interacting agents models). Andersen [1996] interprets the aggregated volatility as the manifestation of numerous heterogeneous information arrivals. Limits to arbitrage, market psychology, heuristics and biases, which are subject of behavioral finance, can also be helpful to explain empirical evidence [see Barberis and Shleifer, 2003].

A number of analytically solvable models were proposed to explore the dynamics of financial market with heterogeneity coming from boundedly rational beliefs of investors about future returns. Brock and Hommes [1998] proposed a model, where investors switch between a number of strategies according to expected or realized excess profits. Stylized simple strategies describe patterns in investors' behavior that are commonly observed empirically - chartism and trend-following. Chiarella and He [2001], Anufriev et al. [2006] and Anufriev [2008] studied an artificial market populated with investors, following heterogeneous strategies and maximizing the expected CRRA utility. Compared to earlier studies that use CARA utilities, they make investment decisions depend on wealth, which is undoubtedly more realistic but technically more difficult. Vanden [2005] introduces a more sophisticated step-wise dependence of the risk aversion on wealth and finds that this can have important consequences for return dynamics. Recently Weinbaum [2009] showed that heterogeneous risk preferences and risk sharing can be the source of volatility clustering.

To our knowledge, all the above-mentioned models of heterogeneity ignore one of its important sources, which is different investment scales. By investment scales we mean typical periods between two consecutive adjustments of investment portfolio, peculiar to a certain type of investors. The heterogeneity of the market with respect to agents' operations frequencies is further referred to as the Multiple Investment Scales (MIS) hypothesis. We suppose that investors maximize expected utility of wealth at the end of some investment period. We call the typical length of this period as investment horizon (or scale).

Earlier the effect of heterogeneity in investment horizons was studied in Anufriev and Bottazzi [2004]. They derive a fixed point for the price of the risky asset dynamics under the assumption that agents maximize expected CARA utility over different periods in future. But their model disregards the effect of various frequencies of portfolio adjustments and, due to the constraints of the CARA assumption, does not realistically account for the dynamics of wealth. They conclude that heterogeneity of investment horizons alone is not enough to guarantee the instability of the fundamental price and the emergence of the non-trivial price dynamics, such as volatility clustering or serial correlations. In this paper we derive the opposite conclusion, which is close to that obtained in Chauveau and Topol [2002]. Working in a different framework, they explained volatility clustering of OTC exchange rates by market microstructure effects, unifying intraday and interday dynamics.

Though not examining the MIS hypothesis analytically, several earlier stud- 
ies evoke the heterogeneity of investment horizons as a possible explanation of the stylized facts in stock price volatility. The assumption that price dynamics is driven by actions of investors at different horizons serves as a micro-economic foundation of the volatility models in Müller et al. [1997]. They suppose that there exist volatility components, corresponding to particular ranges of stock price fluctuation frequencies, that are of unequal importance to different market participants. These participants include intraday speculators, daily traders, portfolio managers and institutional investors, each having a characteristic time of reaction to news and frequency of operations on the market. So frequencies of price fluctuations depend on the periods between asset allocation decisions, and/or the frequencies of portfolio readjustments by investors.

An important question, answered in this paper, is whether the presence of (i) contrarian and trend-following investors and (ii) heterogeneous information arrivals on the market are necessary properties for an interacting agents model to reproduce the stylized facts of the return volatility dynamics. We show that, under some conditions, volatility clustering can arise even in an economy populated with fundamentalist traders only, given that they adjust their portfolios with different frequencies. We also propose a study of the joint effect of the MIS hypothesis and of the bounded rationality in investment strategies.

The rest of the paper is organized as follows. In the next section we introduce the general setting of the model. Section 3 describes the equilibria in the onescale model with boundedly rational investors, re-examining the conclusions of Anufriev et al. [2006] and preparing the ground for the study of the multi-scale case. In section 4 we derive the equilibrium in the MIS case and establish the properties of the return dynamics. In section 5 we illustrate our findings with simulation examples. In conclusion the main results are summarized and possible model extensions are discussed.

\section{A Model for Joint Dynamics of Stock Price and Wealth with Multiple Investment Scales}

In this section we formulate the model and then discuss its various possible specifications and assumptions. The general setup follows the lines of Chiarella and He [2001] and Anufriev et al. [2006], to which we add the MIS hypothesis and some constraints on investors' behavior, discussed later. Where possible, we keep the same notation as in Anufriev et al. [2006], to enable the easy comparison of results.

Consider a two-assets market where $N$ agents operate at discrete dates. The risk-free asset yields a constant positive interest $R_{f}$ over each period and the risky asset pays dividend $D_{t}$ at the beginning of each period. The price of the risk-free asset is normalized to one and its supply is absolutely elastic. The quantity of the risky asset is constant and normalized to one, while its price is determined by market clearing by a Walrasian mechanism. The Walrasian assumption means that all agents determine their demand for the risky asset taking the price of the risky asset $P_{t}$ as parameter. In other, though this price is unobserved at the moment when investors form their demand, they calculate the demand for the risky asset at every possible price and submits this to a hypothetical Walrasian auctioneer. The price is then set so that the total demand 
across all agents equals to one.

The demand of the risky asset is formulated in terms of the shares of wealth of agents, so that $x_{t, i}$ stands for the share of wealth that investor $i$ with wealth $W_{t, i}$ wishes to invest in the risky asset. The corresponding number of units of the asset is $\frac{W_{t, i} x_{t, i}}{P_{t}}$. The market clearing condition imposes:

$$
\sum_{i=1}^{N} x_{t, i} W_{t, i}=1
$$

The wealth of each investor evolves according to the below equation:

$$
\begin{gathered}
W_{t, i}=\left(1-x_{t-1, i}\right) W_{t-1, i}\left(1+R_{f}\right)+\frac{x_{t-1, i} W_{t-1, i}}{P_{t-1}}\left(P_{t}+D_{t}\right)= \\
\left(1-x_{t-1, i}\right) W_{t-1, i}\left(1+R_{f}\right)+x_{t-1, i} W_{t-1, i}\left(1+R_{t}+\varepsilon_{t}\right),
\end{gathered}
$$

where $D_{t}$ is a dividend payment, whose ration to price is supposed to be an iid random variable $\varepsilon_{t}$, and $R_{t}$ is the return on the risky asset. We define the total return by

$$
Y_{t}=\frac{P_{t}+D_{t}}{P_{t-1}} .
$$

Following Anufriev et al. [2006], we rewrite the model in rescaled terms which allows to eliminate the exogenous expansion due to the risk-free asset growth from the model:

$$
w_{t, i}=\frac{W_{t, i}}{\left(1+R_{f}\right)^{t}}, p_{t}=\frac{P_{t}}{\left(1+R_{f}\right)^{t}}, e_{t}=\frac{\varepsilon_{t}}{1+R_{f}}, y_{t}=\frac{Y_{t}}{1+R_{f}} .
$$

By consequence, the rescaled return on the risky asset is defined by:

$$
r_{t}=\frac{p_{t}}{p_{t-1}}-1=\frac{1+R_{t}}{1+R_{f}}-1=\frac{R_{t}-R_{f}}{1+R_{f}} .
$$

In these terms the whole system dynamics simplifies to:

$$
\begin{aligned}
p_{t} & =\sum_{i} x_{t, i} w_{t, i}, \\
w_{t, i} & =w_{t-1, i}\left[1+x_{t-1, i}\left(r_{t}+e_{t}\right)\right] .
\end{aligned}
$$

Proposition 2.1. The rescaled price dynamics, solving the dynamic system (2), verifies:

$$
p_{t}=p_{t-1} \frac{\sum_{i} w_{t-1, i}\left(x_{t, i}-x_{t-1, i} x_{t, i}\right)+e_{t} \sum_{i} x_{t, i} x_{t-1, i} w_{t-1, i}}{\sum_{i} w_{t-1, i}\left(x_{t-1, i}-x_{t, i} x_{t-1, i}\right)},
$$

Proof. See Anufriev et al. [2006].

Proposition 2.1 describes the equilibrium price dynamics in the sense that at each period $t$ Walrasian equilibrium is achieved on our two-asset market. It is straightforward to see that the equilibrium return must satisfy:

$$
r_{t}=\frac{\sum_{i} w_{t-1, i}\left(x_{t, i}-x_{t-1, i}+x_{t, i} x_{t-1, i} e_{t}\right)}{\sum_{i} w_{t-1, i}\left(x_{t-1, i}-x_{t, i} x_{t-1, i}\right)},
$$


if the rescaled return is defined by (1). Note that equation (3) explicitly specifies the return $r_{t}$ conditionally to the information set at period $t-1$, if and only if we impose additional assumptions: both the demand $x_{t, i}$ and the dividend yield $e_{t}$ must be independent of the current price level $p_{t}$.

The simplest assumption about dividends one can suggest to make the model in (2) tractable, is that the dividend yield is an iid non-negative stochastic process. Following Chiarella and He [2001] and Anufriev et al. [2006], we stick to this assumption, though we are aware of the constraints it imposes. Dividends in our economy are deprived of their own dynamics, but follow the risky asset price. Roughly speaking, the amount of dividends available is supposed to automatically adapt to the fluctuations of the price level, so that the mean dividend yield remains unchanged. In real life dividends are paid by stock issuers and so depend on companies' profits and decided payout ratios. If the supply of the risky asset is fixed, one can hardly expect a perfectly linear dependence between average dividends and prices, though a positive relationship between them does exist. However, for the purposes of our paper, the iid assumption for the dividend yield is sufficient.

So far, nothing has been said about the way agents determine the desired proportions of investment in the risky asset. The MIS hypothesis, studied in this paper, implies that some investors do not trade at all time periods and remain passive. During the period, when some investor is out of the market, his share of investment in the risky asset is no longer a result of his decisions but a consequence of price and wealth movements, independent of his will. The following proposition specifies the way investment shares evolve.

Proposition 2.2. Let $x_{t, i}^{-k}$ be the share of investment in the risky asset of investor $i$, who actually participated in the trade $k$ periods ago, $k=1, \ldots, h$ with $h$ being his investment horizon. The investment share verifies the following recurrent relationship:

$$
x_{t, i}^{-k}=\frac{x_{t-1, i}^{-k+1}\left(1+r_{t}\right)}{1+x_{t-1, i}^{-k+1}\left(r_{t}+e_{t}\right)}
$$

Proof. See Appendix.

At the period when investor $i$ readjusts his portfolio, his demand for the risky asset $x_{t, i}^{0}$ is determined according to some investment function. In this paper, we suppose that investment functions are given as the dependence of the share of wealth, invested in the risky asset, on the beliefs about future gains. We also suppose that investment functions are deterministic and do not change over time for the same investor ${ }^{1}$. The beliefs are based on the past observations of prices and dividends, without any private information that could be used to forecast future returns. Moreover, each investment function is supposed to be independent of the current wealth, which is a natural assumption in the CRRA framework. So investor $i$ 's function reads:

$$
x_{t, i}^{0}=f_{i}\left(r_{t-1}, \ldots, r_{t-L_{i}}, e_{t-1}, \ldots, e_{t-L_{i}}\right)
$$

where $L_{i}$ is the maximum lag for historical observations used by the agent $i$, which can be finite or infinite.

\footnotetext{
${ }^{1}$ Note that this does not exclude functions, corresponding to investment strategies that evolve according to predefined rules.
} 
In this paper we will in particular focus on the case of preferences that corresponds to the maximization of the mean-variance CRRA expected utility of wealth ${ }^{2}$. Let us suppose that investors, possibly operating over different time scales, maximize a mean-variance expected utility:

$$
\max _{x_{t, i}^{0}}\left\{\mathrm{E}_{t-1, i}\left(W_{t+h, i}\right)-\frac{\gamma_{i}}{2 W_{t, i}} \operatorname{Var}_{t-1, i}\left(W_{t+h}\right)\right\}
$$

with operators $\mathrm{E}_{t-1, i}(\cdot)$ and $\operatorname{Var}_{t-1, i}(\cdot)$ standing for the beliefs of agent $i$ about the mean and variance given the information at time $t-1$. The information set of period $t-1$ includes the prices of the risky asset and the dividends at time $t-1$ and earlier. The coefficient $\gamma_{i}$ is a positive constant that measures the risk aversion of investor $i$. The time horizon of decision taking, denoted $h$, corresponds to the period of time when investor $i$ does not readjust his portfolio. The number of units of risky asset in investor's possession remains constant over $[t ; t+h]$, while the share of investment in the risky asset may evolve. We assume that dividends, paid by the risky asset during this period, are accumulated on the bank account, yielding the risk-free rate.

Proposition 2.3. The solution $x_{t, i}^{0 *}$ of the maximization problem (6) is approximately given by:

$$
x_{t}^{0 *} \approx \frac{\mathrm{E}_{t-1, i}\left[\sum_{k=1}^{h}\left(e_{t+k}+r_{t+k}\right)\right]}{\gamma_{i} \operatorname{Var}_{t-1, i}\left[\sum_{k=1}^{h}\left(e_{t+k}+r_{t+k}\right)\right]}
$$

Proof. See Appendix.

Chiarella and He [2001] show that the expression (7) with $h=1$ also emerges as an approximative solution in the maximization problem with the power utility function. This approximation, however, consists in a discretization of a continuous-time process with Gaussian increments and thus it can be far from the real solution for non-infinitesimal time units. So we prefer to work with mean-variance maximization directly. Alternatively, an investment function of the form (7) could be set on an a priori basis since it describes the behavior of a mean-variance investor with constant relative aversion to risk.

Notice that if the return process is iid, $\mathrm{E}_{t-1}\left[y_{t, t+h}\right]=h \mathrm{E}_{t-1}\left[r_{t+1}+e_{t+1}\right]$ and $\operatorname{Var}_{t-1}\left[y_{t, t+h}\right]=h \operatorname{Var}_{t-1}\left[r_{t+1}+e_{t+1}\right]$. This ensures that if, in addition, risk aversion is homogeneous for investors at all scales $\left(\gamma_{i}=\gamma\right)$, the demand for the risky asset does not depend on the investment horizon. We maintain the assumption of homogeneous risk aversion throughout this paper.

In equation (7) the share of wealth to be invested in the risky asset depends exclusively on the beliefs of agents about future yields. In the heterogeneous agents literature these beliefs are based on historical prices of the risky asset up to a certain lag. Following Chiarella and He [2001], we do not include $p_{t}$ in the information set for beliefs formations in order to avoid unnecessary complexity. Nevertheless, in the MIS case the aggregate demand on the risky asset naturally depends on the current price level. Indeed, suppose that the previous date, when

\footnotetext{
${ }^{2}$ Most of our results are valid also in the case of the general investment function (5), not necessarily representing the beliefs about mean and variance. This will be specially indicated further in the paper.
} 
investor $i$ participated in the trade, was $t-k$ and that at this date the share of wealth $x_{t-k, i}^{0}$ he invested in the risky asset was determined according to (5). Then it follows from (4) that his current investment share $x_{t, i}^{-k}$ depends on the historical returns and dividend yields up to the lag $L_{i}+k-1$, but also on the current return and the dividend yield, which are unknown before the trade at date $t$. So equation (3) does not explicitly specify the dynamics of the risky return.

In the following section we study the dynamics of the price and wealth in the model with one scale, which is a particular case of the model, introduced in the previous section. We further refer to it as the benchmark model. We extend the analysis of Anufriev et al. [2006] in several aspects, also important in the MIS case, studied later.

\section{Equilibria in the One-Scale Model with Bounded Rationality}

As we have mentioned before, in the one-scale case, equation (3) completely and explicitly describes the dynamics of the return on the risky asset under the market clearing condition. By specifying the demand function, one can determine the equilibrium price and return. This equilibrium dynamics was earlier studied in Anufriev et al. [2006], who replace the actual dividend yield by its mean and work with the so-called "determenistic skeleton" of the system. In the deterministic case the (rescaled) return is constant: $r_{t}=r$. The authors prove that two types of equilibria are possible: either a single agent survives ${ }^{3}$, or many agents survive, but in both cases the equilibrium share of investment in the risky asset and the steady growth rate of its price are determined in a similar way. They must satisfy the relationship, which is easily obtained from (2) for a single-agent case, when we set $x_{t}=x_{t-1}$ for all $t$. This relationship is called the Equilibrium Market Line (EML) and reads:

$$
x=\frac{r}{r+\bar{e}}
$$

where $\bar{e}$ is the mean dividend yield.

The demand functions of investors depend on a single variable and are of the form:

$$
x=f\left(r_{t-1}, \ldots, r_{t-L}\right)=f(r, \ldots, r)=\tilde{f}(r)
$$

The equilibrium points are determined as the intersections of the demand curve $\tilde{f}(r)$ and the EML. It is shown that, if multiple agents survive, their demand functions must all intersect the EML at the same equilibrium point. Stability conditions, depending on the properties of derivatives of $f_{i}(\cdot)$ with respect to returns at different lags, are established. We refer the reader to the original paper of Anufriev et al. [2006] for further details.

In our approach, the main difference is that we are interested in the stochastic properties of the return process. In particular, we establish analytically, under what conditions the dynamics of returns is "simple" (iid) and when it displays "interesting" dynamic patterns (conditional heteroscedasticity and/or

\footnotetext{
${ }^{3}$ i.e. his share in the total wealth does not decrease to zero in infinite time
} 
serial correlations). In our view, this type of approach is appropriate for the study of boundedly rational behavior of agents, whose investment functions are based on beliefs about mean and variance of the return process. This point is explained further.

For the case of multiple agents with heterogeneous investment functions, Anufriev et al. [2006] determine which form of the demand function "dominates" the others. For example, if a trend-follower (investor who strongly extrapolates past returns) meets a fundamentalist (investor, whose demand function is independent of the price history), we can predict which of them survives, depending on the respective form of their investment functions. Under some conditions, the trend-follower outperforms the fundamentalist and survives. A striking feature of the model is that equilibria are possible for almost any, and even completely senseless, demand functions and can even be stable.

The problem here is with bounded rationality. More precisely, it is important to what extent the rationality is bounded. In Anufriev et al. [2006] and Chiarella and $\mathrm{He}$ [2001], investment functions are given a priori, and though they formally depend on the beliefs of agents about the mean and variance of future returns, there are no constraints on how these beliefs should be related to the true quantities.

Bounded rationality means that agents may not know the true model. But in equilibrium, when the return on the risky asset is supposed to be constant, it is hard to admit that the beliefs have nothing to do with reality. Besides, the stability of such equilibria hardly makes sense from the economic point of view, since agents would have incentives to change their strategies, if they were allowed to.

In Brock and Hommes [1998] agents are allowed to switch between strategies, according to the profits they yield in the past. The agents can thus be claimed to be procedurally rational, because they try to rationally choose strategies according to some criteria. In our case, a more exact definition of procedural rationality can be helpful to study the model analytically. We restrain the class of admissible investment functions, considerably reducing the possibilities for non-rationality of economic agents, without necessarily imposing rational expectations.

Investment functions basically define how agents' beliefs about future returns are formed, i.e. they are concise descriptions of the outputs of the beliefs-making procedure. The rationality of such procedure can be tested in some simple reference case, where the outcome of the procedure is expected to correspond to the rational behavior. In our case of mean-variance investor, we require that for the iid returns beliefs about mean and variance of the process should be unbiased. This is formalized in the following definition.

Definition 3.1. An investment function of the form:

$$
x_{t, i}^{0}=\tilde{f}_{i}\left(\mathrm{E}_{t-1, i}\left[y_{t, t+h}\right], \operatorname{Var}_{t-1, i}\left[y_{t, t+h}\right]\right)
$$

is called procedurally rational if the beliefs $\mathrm{E}_{t-1, i}\left(y_{t-1, t+h}\right)$ and $\operatorname{Var}_{t-1, i}\left(y_{t, t+h}\right)$ about the mean and variance of the future total returns are unbiased estimates of these quantities with finite error, if the true process $y_{t, t+1}$ is iid.

This definition is an adaptation of Simon's procedural rationality ${ }^{4}$ to our

\footnotetext{
4 "Behavior is procedurally rational when it is the outcome of appropriate deliberations. Its procedural rationality depends on the process that generated it" [Simon, 1976, p.131].
} 
context. It basically states that, if previous observations of returns display no non-trivial dynamic patterns, the beliefs about mean and variance of investors should have no systematic error. Note that in no way we state that returns should actually follow an iid process. We only describe the behavior of the investment function in this hypothetical case, in order to impose some constraints on the "reasonability" of the decision taking procedure used by investors.

Note that our definition does not contradict to the concept of bounded rationality, but it requires some moderate degree of consistency in investors' beliefs. Procedurally rational investors can actually be trend-followers or contrarians. Consider, for example, the following specifications for the beliefs about the mean of future returns:

$$
\begin{array}{r}
\mathrm{E}_{t, i}\left(y_{t+1}\right)=c_{i}+\frac{d_{i}}{l} \sum_{k=1}^{l} y_{t-k} \quad(A) \\
\mathrm{E}_{t, i}\left(y_{t+1}\right)=\frac{1-d_{i}}{L} \sum_{k=1}^{L} y_{t-k}+\frac{d_{i}}{l} \sum_{k=1}^{l} y_{t-k}
\end{array}
$$

The function of the type, analogous to (11A), is used in Chiarella and He [2001] to represent the behavior of heterogeneous investors. Here $c_{i}$ is some constant that represents the risk premium, required by the investor, and $d_{i}$ is a behaviorist parameter, which specifies, how investor $i$ extrapolates the performance of the risky asset over $l$ recent periods. If $d_{i}=0$, the investor is fundamentalist, if $d_{i}>$ 0 he is a trend-follower, otherwise contrarian (chartist). It is easy to see that this specification does not correspond to our definition of the procedural rationality, unless simultaneously $c_{i}=0$ and $d_{i}=1$. The function (11B) also allows for the extrapolation of the recent returns via the parameter $d$. If $l<L$, positive $d_{i}$ corresponds to the trend-following. But this function verifies our condition for procedural rationality: in the iid case the expectation of the difference in the short-term and the long-term mean is null.

Suppose that investors' preferences are described by the mean-variance function of the form (7), satisfying definition 3.1. Having restrained the set of admissible investment functions, we turn to the study of the price dynamics in the benchmark model. In the following theorem we establish the conditions that must be verified by the investment function to ensure "simple" dynamics of the returns, which can be associated with some steady growth trajectory. It states that the assumption of investors' rational expectations is equivalent to the iid dynamics of returns.

Theorem 3.2. In the benchmark model with homogeneous procedurally rational agents the return process can be iid with finite mean and variance if and only if investors have rational expectations. In this case the mean and variance of the return process are uniquely defined by the mean dividend yield and investors' risk aversion.

Proof. The homogeneity of agents means that they all have the same investment functions and, in particular, the same risk aversion $\gamma_{i}=\gamma$. In the benchmark model they also use the same information, so $x_{t, i}=x_{t, j}, \forall t, i, j$ and we can drop the second subscript. Thus this case is analogous to a single-agent model with a representative agent. Simplifying (2), it is straightforward to see that the 
returns do not directly depend on the wealth dynamics, since we have:

$$
r_{t}=\frac{x_{t}-x_{t-1}+e_{t} x_{t} x_{t-1}}{\left(1-x_{t}\right) x_{t-1}}
$$

If $r_{t}$ is an iid process, then $r_{t}$ is independent of the returns' history $r_{t-1}, r_{t-2}, \ldots$, but it is also independent of $x_{t}, x_{t-1}, \ldots$ since the latter depend only on past returns. Consider the stochastic process $r_{t \mid t-1}$ of returns, conditional to the information at period $t-1$, which is defined as the set $I_{t}=\left\{r_{t-1}, r_{t-2}, \ldots ; x_{t}, x_{t-1}, \ldots\right\}$. It follows from the above that this process is also iid.

The quantities $x_{t \mid t-1}$ and $x_{t-1 \mid t-1}$ are both deterministic since the investment function at time $t$ depends only on returns at time $t-1$ and earlier. So the conditional mean and variance of returns are:

$$
\begin{gathered}
\mathrm{E}_{\mathrm{t}-1}\left(r_{t}\right)=\frac{x_{t}-x_{t-1}+\bar{e} x_{t} x_{t-1}}{\left(1-x_{t}\right) x_{t-1}} \\
\operatorname{Var}_{\mathrm{t}-1}\left(r_{t}\right)=\sigma_{e}^{2} \frac{x_{t}^{2}}{\left(1-x_{t}\right)^{2}}
\end{gathered}
$$

with $\bar{e}$ and $\sigma_{e}^{2}$ the mean and variance of the dividend yield process respectively (both are supposed to be constant). Note that here the operators $\mathrm{E}_{\mathrm{t}}(\cdot)$ and $\operatorname{Var}_{\mathrm{t}}(\cdot)$ no longer refer to the agent's beliefs, but to the mathematical expectation and variance of random variable.

We have shown that the process $r_{t \mid t-1}$ is iid. Then it follows from (13) that $x_{t}=x_{t-1}=x^{*}$ and equation (12) simplifies to:

$$
r_{t}=e_{t} \frac{x^{*}}{1-x^{*}}
$$

The investment function $f\left(r_{t-1}, \ldots, r_{t-L}\right)$ takes the value $x^{*}$ with probability 1 for all values $r_{t-1}, \ldots, r_{t-L}$ drawn from an iid process if and only if it is a constant function in any domain where the vector $r_{t-1}, \ldots, r_{t-L}$ takes values with non-zero probability.

Since the return dynamics, given by (15), is iid, procedural rationality implies that the beliefs of investors are unbiased:

$$
\begin{aligned}
\mathrm{E}_{t-1}\left[r_{t+1}+e_{t+1}\right] & =\bar{e} \frac{x^{*}}{1-x^{*}}+\bar{e}=\frac{\bar{e}}{1-x^{*}} \\
\operatorname{Var}_{t-1}\left[r_{t+1}+e_{t+1}\right] & =\operatorname{Var}_{t-1}\left[\frac{e_{t+1}}{1-x^{*}}\right]=\frac{\sigma_{e}^{2}}{\left(1-x^{*}\right)^{2}}
\end{aligned}
$$

Then, according to (7) with $h=1$, the investment share satisfies:

$$
x^{*}=\frac{\frac{\bar{e}}{1-x^{*}}}{\gamma \frac{\sigma_{e}^{2}}{\left(1-x^{*}\right)^{2}}}
$$

From (17) we obtain a unique solution for $x^{* 5}$ :

$$
x^{*}=\frac{\bar{e}}{\gamma \sigma_{e}^{2}+\bar{e}}
$$

\footnotetext{
${ }^{5}$ Analoguous computation in terms of not-rescaled variables gives $x^{*}=$ $\mathrm{E}_{t-1}\left[\varepsilon_{t+1}\right]\left(\gamma \operatorname{Var}_{t-1}\left[\varepsilon_{t+1}\right]+\mathrm{E}_{t-1}\left[\varepsilon_{t+1}\right]\right)^{-1}$, which is slightly different from (18) because of the first order approximation. This difference is of no incidence in our context.
} 
This proves that if returns are iid, then the investment share $x^{*}$, computed from (18), uniquely determines the mean and variance of the process $r_{t \mid t-1}$ (or, in other words, the necessary conditions for the iid return dynamics). It is easy to see that the solution we derived corresponds to the case where investors have rational expectations.

It can be shown straightforwardly, that these conditions are also sufficient. It suffices to plug the constant $x^{*}$ in the equation (12) for returns and then verify that the expectation and variance of returns are constant and given by (13) and (14) respectively.

An important consequence of theorem 3.2 is that, in the benchmark model with homogeneous procedurally rational investors, returns on the risky asset never have simple iid dynamics, unless the investors have rational expectations. Note that equation (14) describes conditional volatility dynamics in the model. It follows from (14) that for $0<x_{t}<1$, conditional variance always increases with $x_{t}$. If the investment function depends positively on the historical mean of returns and negatively on their historical variance (which is an appropriate assumption in a procedurally rational context), then the conditional variance is a decreasing function of historical variance and increasing function of historical returns. At the same time, volatility has the same "memory" as the squared share of investment in the risky asset, which is determined by investors' beliefs. If the latter are adjusted slowly, then volatility also adjusts slowly.

Now consider the return dynamics in a more general case, when homogeneous agents having arbitrary (not necessarily boundedly rational) investment functions of the form

$$
x_{t}=f\left(r_{t-1}, \ldots, r_{t-L}, e_{t-1}, \ldots, e_{t-L}\right) .
$$

The stochastic process (12) for the return dynamics is non-linear. We study the properties of its first-order Taylor linearization in the neighborhood of the expected return, $r_{t-k}=\bar{r}$ for all $k$, with arbitrary values of $e_{t-k}$. We denote $\widetilde{e}_{t}=e_{t}-\bar{e}$ and $\widetilde{r}_{t}=r_{t}-\bar{r}$ the deviations of dividend yield and return from their average values. We also denote $f_{k}^{\prime}$ the first derivative of $f(\cdot)$ with respect to $r_{t-k}$ for $k=1, \ldots, L$. The form of the return process is given by the following theorem.

Theorem 3.3. In the benchmark model with homogeneous agents, if the return process is covariance stationary, it satisfies:

$$
\begin{aligned}
r_{t} & =\frac{\bar{x}}{1-\bar{x}} \bar{e}+\widetilde{r}_{t} \\
\widetilde{r}_{t} & =\sum_{k=1}^{L+1} a_{k} \widetilde{r}_{t-k}+v_{t} \widetilde{e}_{t} \\
v_{t} & =\frac{\bar{x}}{1-\bar{x}}+\sum_{k=1}^{L} b_{k} \widetilde{r}_{t-k}
\end{aligned}
$$


with:

$$
\begin{aligned}
a_{1} & =\frac{f_{1}^{\prime}[1-\bar{x}(1-\bar{e})]}{\bar{x}(1-\bar{x})^{2}} \\
a_{k} & =\frac{f_{k}^{\prime}[1-\bar{x}(1-\bar{e})]+f_{k-1}^{\prime}(\bar{x}-1)}{\bar{x}(1-\bar{x})^{2}}, k \in\{2, \ldots, L\} \\
a_{L+1} & =\frac{f_{L}^{\prime}}{\bar{x}(1-\bar{x})} \\
b_{k} & =\frac{f_{k}^{\prime}}{(1-\bar{x})^{2}}, k \in\{1, \ldots, L\} \\
\bar{x} & =
\end{aligned}
$$

Proof. See Appendix.

Equations (19) can be written in the equivalent form:

$$
\begin{aligned}
\widetilde{r}_{t} & =\sum_{k=1}^{L+1} a_{k} \widetilde{r}_{t-k}+\sigma_{e}\left(u_{t}+\frac{\bar{x}}{1-\bar{x}}\right) \varepsilon_{t} \\
u_{t}^{2} & =\sum_{k=1}^{L} b_{k}^{2} \widetilde{r}_{t-k}^{2}+2 \sum_{\substack{i, j \in\{1, \ldots, L\} \\
i \neq j}} b_{i} b_{j} \widetilde{r}_{t-i} \widetilde{r}_{t-j}
\end{aligned}
$$

with $\varepsilon_{t}$ a standardized independent white noise. This stresses the ARCH-like nature of the returns process. Note that its mean is described by an expression, equivalent to the definition of equilibrium on the EML in [Anufriev et al., 2006].

Now we can turn to the case with heterogeneous agents, i.e. the case when $x_{t, i}$ are determined in a different way by each investor. Again we restrict the investment functions to mean-variance and procedurally rational. Theorem 3.4 shows that the simple iid dynamics does not appear generically if investors are heterogeneous.

Theorem 3.4. In the benchmark model with heterogeneous procedurally rational agents the return process can be iid with finite mean and variance only if the aggregate share of wealth invested in the risky asset is constant. In this case the mean and variance of the return process are proportional to the mean and variance of the dividend yield.

Proof. See Appendix.

Basically this theorem says that if the aggregate share of investment in the risky asset is subject to stochastic shocks or fluctuations, the return dynamics is almost surely not trivial and displays dynamic patterns. The situation, when the aggregate investment function is constant and returns are iid, can arise only when the dependence of the individuals' procedurally rational investment functions on the past returns is not characterized by prevailing patterns. More precisely, individual deviations $\nu_{t, i}=x_{t, i}-\bar{x}$ from some constant investment share $\bar{x}$, weighted by the wealth portions of agents $\xi_{t, i}$, are eliminated by aggregation with probability one:

$$
P\left(\sum_{i=1}^{N} \xi_{t, i} \nu_{t, i}=0\right)=1
$$


for all $t$. For this condition to be true, some form of the law of large numbers must be satisfied and, moreover, the expectation of $\nu_{t, i}$, conditional on past returns, must be constant. This is improbable in the situation, when all investors base their expectations on the same vector of realized past returns and this vector is not constant.

\section{Equilibria with Multiple Investment Scales}

In the previous section we considered the case when investors have the same investment horizons, but possibly different investment functions. Now we come back to th MIS hypothesis and study another source of heterogeneity, related to investment horizons. Now assume that there exist $H$ investment scales with portfolio readjustment periods $h=1, \ldots, H$ time units, so that each agent has a characteristic investment scale that does not change. Suppose that within each investment scale investors are homogeneous, i.e. have the same specifications of demand function. Finally, suppose that at each date the wealth of investors, having the same investment scale, is distributed so that a constant part of this wealth, equal to $1 / h$ belongs to the investors, rebalancing their portfolios at the current date.

The latter assumption does not necessarily imply that the wealth can be redistributed between different groups of investors in a given period. Rather, it means that there is a large number of investors, going in and going out, and they have random dates of intervention on the market but fixed frequencies of trades. So the composition of each cohort of investors may change, but its average share of wealth remains constant.

Under these simplifying assumptions, we can aggregate all investors, acting at the same scale $h$, and replace them by a representative agent, whose share of wealth, invested in the risky asset, satisfies:

$$
x_{t, h}=\frac{1}{h} \sum_{k=0}^{h-1} x_{t}^{-k}
$$

Equations (2), describing the dynamics of the system, are still true, but now the subscript $i$ corresponds to the investment scale and the wealth $w_{t, i}$ is the aggregate wealth of a class of investors, having the same investment horizon. In section 2 we derived equation (4) that describes the evolution of the share of investor's wealth, invested in the risky asset, when he does not trade. Then the complete system of equations, describing the dynamics of risky return, reads:

$$
\begin{aligned}
r_{t} & =\frac{\sum_{h=1}^{H} w_{t-1, h}\left(x_{t, h}-x_{t-1, h}\right)+e_{t} \sum_{h=1}^{H} x_{t, h} x_{t-1, h} w_{t-1, h}}{\sum_{h=1}^{H} w_{t-1, h} x_{t-1, h}\left(1-x_{t, h}\right)} \\
x_{t, h} & =\frac{1}{h} \sum_{k=0}^{h-1} x_{t, h}^{-k} \\
x_{t, h}^{-k} & =\frac{x_{t-1, h}^{-k+1}\left(1+r_{t}\right)}{1+x_{t-1, h}^{-k+1}\left(r_{t}+e_{t}\right)}
\end{aligned}
$$

As noted above, an important feature of (23) is that it describes the return dynamics only implicitly, because the investment share for all but the shortest 
scales inevitably depends on current return. The relation between the price and the dividend process becomes non-linear and complicated, because it includes previous dividends. For the general equation of price dynamics, we can prove that:

Theorem 4.1. Whatever the number of scales $H$, there always exists at least one positive market clearing price for which the return $r_{t}$ satisfies (23).

Proof. See Appendix.

It is important to specify conditions, under which the multi-scale dynamics does not degenerate, that is the portions of wealth, held by the agents, investing at each scale, do not tend to zero as time tends to infinity.

More precisely, denote $\xi_{t, h}$ the portion of wealth that belongs to investors of type $h$.

Definition 4.2. The MIS dynamics, described by equation (23), is called nondegenerating, if for any investment scale $h$ such as $\xi_{0, h}>0$ we have :

$$
P\left(\xi_{t, h}=0\right)=0
$$

when $t$ approaches infinity.

In the following theorem we establish the necessary and sufficient conditions that provide for the non-degenerating dynamics in the MIS system. Denote $g_{t, h}$ the growth rate of wealth of investors of type $h$ at time $t$ :

$$
g_{t, h}=\frac{w_{t, h}}{w_{t-1, h}}=1+x_{t-1, h}\left(r_{t}+e_{t}\right)
$$

We suppose that the stochastic process $\ln \left(g_{t, h}\right)$ is covariance-stationary. Furthermore, we suppose that it verifies the following conditions on its memory:

$$
N^{-1}\left\|\left\{\operatorname{Cov}\left(\ln \left(g_{t+i, h}\right), \ln \left(g_{t+j, h}\right)\right)\right\}_{\{i=1, \ldots, N, j=1, \ldots, N\}}\right\|_{2} \leq C
$$

for all positive $N$ and some finite $C$. This technical condition, implying that $\ln \left(g_{t, h}\right)$ is a stochastic process with bounded spectral density, ensures that the average growth rates of wealth converge almost surely to their expectation as time tends to infinity. This result is proved in Ninness [2000].

Theorem 4.3. The multiple investment scales dynamics, described by equation (23), is non-degenerating if and only if for any $h$ :

$$
\mathrm{E}\left[\ln \left(g_{t, i}\right)\right]=\mathrm{E}\left[\ln \left(g_{t, j}\right)\right], \forall i, j \in\{1, \ldots, H\}
$$

Proof. See Appendix.

To interpret the theorem, notice that the log growth rate of the wealth is approximately equal to the product of the total return on the risky asset and the share of wealth, invested in the risky asset at the previous period. Thus, for the model to be non-degenerating, investors should either have the same average share of investment in the risky asset, or lower investment shares should be compensated by positive correlation of the investment share with future return. 
A particular case of the non-degenerating system is non-predictive equal-in-law investment shares:

$$
\begin{aligned}
x_{t, i} & \stackrel{L}{=} x_{t, j} \forall i, j \in\{1, \ldots, H\}, \forall t \\
\operatorname{Cov}\left(x_{t-i, h}, r_{t}\right) & =0, \forall h \in\{1, \ldots, H\}, \forall i, t .
\end{aligned}
$$

Note that in MIS system the existence of autocorrelations in returns implies correlation of the investment shares with the future returns. Moreover, the latter is higher for investors at longer scales, because at each time period there are more passive investors, whose investment shares depend on past returns, even if elementary investment functions are constant. Thus condition (25) is related to the absence of serial correlations in returns.

By analogy with the one-scale case, we analyze the equilibrium dynamics of the system (23). First let us study the "mean" dynamics, supposing $e_{t}=\bar{e}$. The following theorem shows that there exists an equilibrium path $r_{t}=\bar{r}$ that solves the deterministic analog of (23).

Theorem 4.4. The dynamic system (23) with $e_{t}=\bar{e}$ has a unique equilibrium solution with constant return:

$$
\begin{array}{r}
\bar{r}=\frac{\bar{x}}{1-\bar{x}} \bar{e}, \\
f_{h}(\bar{r}, \ldots, \bar{r})=\bar{x}
\end{array}
$$

Proof. Suppose that the system has some equilibrium solution $r=\bar{r}$. This implies:

$$
\frac{\sum_{h=1}^{H} \bar{w}_{t-1, h}\left[\bar{x}_{t, h}-\bar{x}_{t-1, h}+\bar{e} \bar{x}_{t, h} \bar{x}_{t-1, h}\right]}{\sum_{h=1}^{H} \bar{w}_{t-1, h} \bar{x}_{t-1, h}\left(1-\bar{x}_{t, h}\right)}=\bar{r}<\infty
$$

Besides, it is easy to notice that, for any $h$, investment in the risky asset is constant, because the investment functions depend only on the past realizations of returns and dividend yields, equal to $\bar{r}$ and $\bar{e}$ respectively:

$$
\bar{x}_{t, h}=f_{h}(\bar{r}, \ldots, \bar{r}, \bar{e}, \ldots, \bar{e})=\bar{x}_{h} .
$$

At the same time conditions (25) implies that average investment shares are equal for all types of investors. Thus the trajectories of wealth satisfy:

$$
\bar{w}_{t-1, h}=w_{0, h}[1+\bar{x}(\bar{r}+\bar{e})]^{t-1} .
$$

Thus equation (27) simplifies to:

$$
\frac{\bar{e} \bar{x}}{1-\bar{x}}=\bar{r}
$$

which is equivalent to:

$$
\bar{x}=\frac{\bar{r}}{\bar{r}+\bar{e}} .
$$

We need to verify that (28) is compatible with the multi-horizon dynamics of the investment shares, characterized by passiveness of a part of agents at some time periods. Recall that the investment in risky asset of each type of agents $h$ is the mean of investments of agents that readjusted their portfolios with $0, \ldots, h-1$ periods ago. But whenever the readjustment takes place, 
the investment share, depending on lagged returns and dividend yields, always takes the same value $\bar{x}_{h}^{0}$. At the next period after portfolio readjustment the investment share of the passive investor becomes:

$$
\frac{\bar{x}_{h}^{0}(1+\bar{r})}{1+\bar{x}_{h}^{0}(\bar{r}+\bar{e})}
$$

We define the function $g=\Re \rightarrow \Re$ as:

$$
g(x)=\frac{x(1+\bar{r})}{1+x(\bar{r}+\bar{e})}
$$

and $g^{k}(x)$ as a $k$-times composition of function $g(\cdot)$, that is $g \circ g \ldots \circ g(x)$, with $g^{0}(x)$ defined as $g(x)=x$. Then for any $h$ we have:

$$
\bar{x}_{h}=\frac{1}{h} \sum_{k=0}^{h} g^{k}\left(\bar{x}_{h}^{0}\right)
$$

Now notice that

$$
g\left(\frac{\bar{r}}{\bar{r}+\bar{e}}\right)=\frac{\bar{r}}{\bar{r}+\bar{e}},
$$

which implies that $\bar{x}_{h}^{0}=\frac{\bar{r}}{\bar{r}+\bar{e}}$ satisfies equation (30). This proves that if the equilibrium return exists, it satisfies:

$$
\bar{r}=\frac{\bar{e} \bar{x}}{1-\bar{x}},
$$

and thus is uniquely defined.

We can now study the properties of the stochastic process for the risky returns and compare the results with those, obtained for the one-scale case. As before, we will proceed by the linearization of the dynamic system. Define the following function $F: \Re^{t-1} \times \Re^{t-1} \times \Re \times \Re \rightarrow \Re$ :

$$
\begin{gathered}
F\left(r_{1}, \ldots, r_{t-1}, e_{1}, \ldots, e_{t-1}, r_{t}, e_{t}\right)= \\
\frac{\sum_{h=1}^{H} w_{t-1, h}\left[x_{t, h}-x_{t-1, h}+e_{t} x_{t, h} x_{t-1, h}\right]}{\sum_{h=1}^{H} w_{t-1, h} x_{t-1, h}\left(1-x_{t, h}\right)}-r_{t}
\end{gathered}
$$

with $x_{t, h}$ defined as in (23). The following theorem describes the equilibrium dynamics in the neighborhood of the solution of the deterministic analog of the system.

Theorem 4.5. In the model with homogeneous rational agents and multiple investment scales, the return process is approximately described by:

$$
\begin{aligned}
r_{t} & =\bar{r}+\hat{r}_{t}+\bar{V} \widetilde{e}_{t}, \\
\hat{r}_{t} & =\sum_{k=1}^{H-1} A_{k} \hat{r}_{t-k}+V_{t} \widetilde{e}_{t}, \\
V_{t} & =\sum_{k=1}^{H-1} B_{k} \hat{r}_{t-k}
\end{aligned}
$$


where:

$$
\begin{aligned}
A_{k} & =\frac{a_{k}-b_{k}}{(1-c)(1+\bar{r})^{k}}, \\
B_{k} & =\frac{\bar{x}(1-2 \bar{x})\left(c b_{k}-a_{k}\right)}{(1-\bar{x})(1+\bar{r})^{k+1}(1-c)}, \\
\bar{V} & =\frac{\bar{x}}{1-\bar{x}}, \\
a_{k} & =\sum_{h=k+2}^{H} \frac{h-k-1}{h} \xi_{0, h}, \\
b_{k} & =\sum_{h=k+1}^{H} \frac{h-k}{h} \xi_{0, h}, \\
c & =\sum_{h=1}^{H} \frac{h-1}{h} \xi_{0, h} .
\end{aligned}
$$

Proof. See Appendix.

The result of theorem 4.5 shows that the return dynamics in the multiscale model with rational investors is very close to the one-scale dynamics in the rational expectations case, the only difference being the term $\hat{r}_{t}$. It represents the deviation from the hypothetical trajectory of returns, that would be realized in a one-scale market, and can be interpreted as error correction term. Note that there is no constant in the volatility of the disturbance term, which means that the correction term either vanishes or explodes, depending on the values of coefficients $A_{k}, B_{k}$ and the variance of $\widetilde{e}_{t}$. We will study its behavior for plausible values of parameters in the following section. Note that the dynamics in the multiscale case is considerably different from the general one-scale case, described by theorem 3.3. There the terms, containing serial correlations and heteroscedasticity, are not vanishing, while in the multiscale rational case their presence is temporal after a shock, in the absence of which they completely disappear from the return dynamics.

Theorem 4.5 refers to the case, when investors' demand functions at the times of portfolio readjustment are trivial: investment shares are constant at the level, corresponding to the rational equilibrium, which coincides with the one-scale equilibrium. In practice, investment decisions may depend on the historical returns, so the framework of procedural rationality would be more adequate for modeling. One can establish a general analytical representation of the return dynamics in this case. Indeed, equations (A-9), (A-11) and (A-12) in the proof of theorem 4.5 (see Appendix) still hold, but instead of (A-10) we need to adopt a general form for the investment functions, as in theorem 3.3. However, in our view, such general representation would be of little practical value. Instead, using simulation, we explore the return dynamics, corresponding to concrete stylized examples of investment functions. This issue is addressed in the following section. 


\section{Simulation Study}

We determined the equations of the risky return dynamics in the case of the market, populated with rational participants, acting at one and several investment horizons. We also established the framework for the study of the procedurally rational investment, that can incorporate behavioral patterns, such as trend extrapolation and contrarian strategies. Our goal in this section is to explore the empirical properties of the return series, generated by different versions of our model, and to associate the properties of the model with the stylized patterns, observed on real market data: contrarian returns, trend formation and conditional heteroscedasticity.

From observation of (32) it is clear that introducing multiple scales changes the way, in which the dynamic system for the risky return reacts to shocks. These shocks could be of completely exogenous or of behavioral nature. We will first study the case when, along with the "normal" disturbance term, interpreted as dividend yield, the model is occasionally perturbated by exogenous shock on returns, unrelated to the investment functions. Such abnormal returns can reflect deviations from market clearing equilibrium at some time periods.

Returns trajectories are simulated for a market with five horizons, where abnormal returns occur at random periods, on average once per 50 trades. We are interested in the values of coefficients $A_{k}$ and $B_{k}$, that determine the way the shock at period $t$ is reverberated at future dates. Note that in a one-scale model such shocks have absolutely no incidence on future returns. The abovementioned coefficient depend on $a_{k}, b_{k}$ and $c$, that characterize how initial wealth is distributed among investors. 
Figure 1: Shocks to returns, $\cap$-shape wealth distribution

(a) Model Parameters
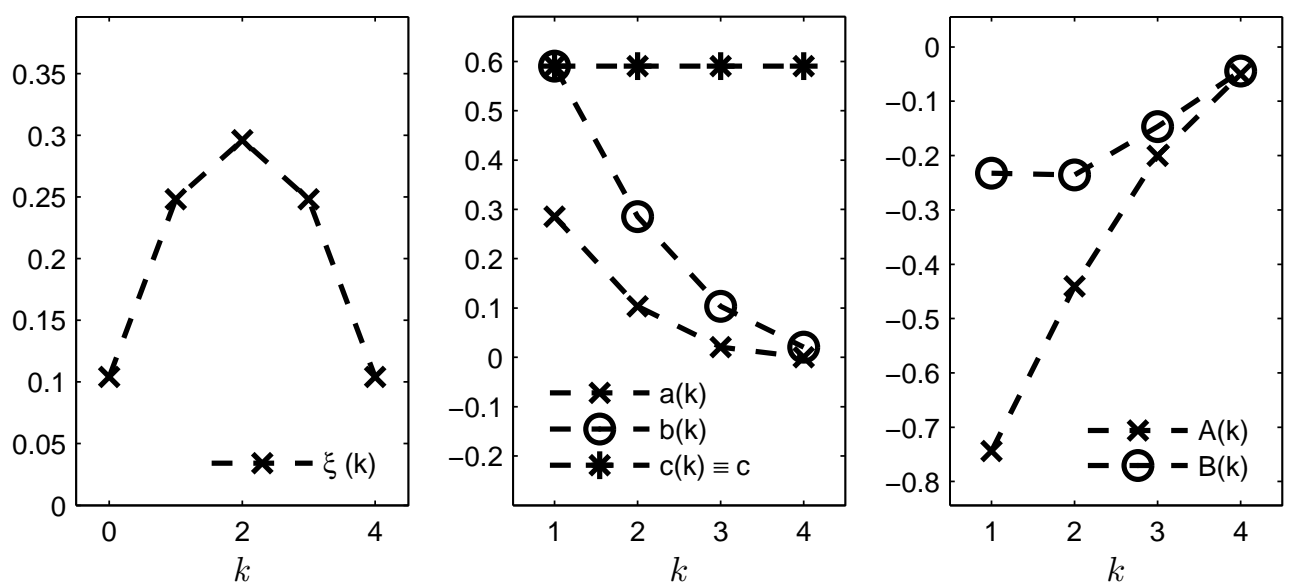

(b) Reaction to Exogenous Shocks
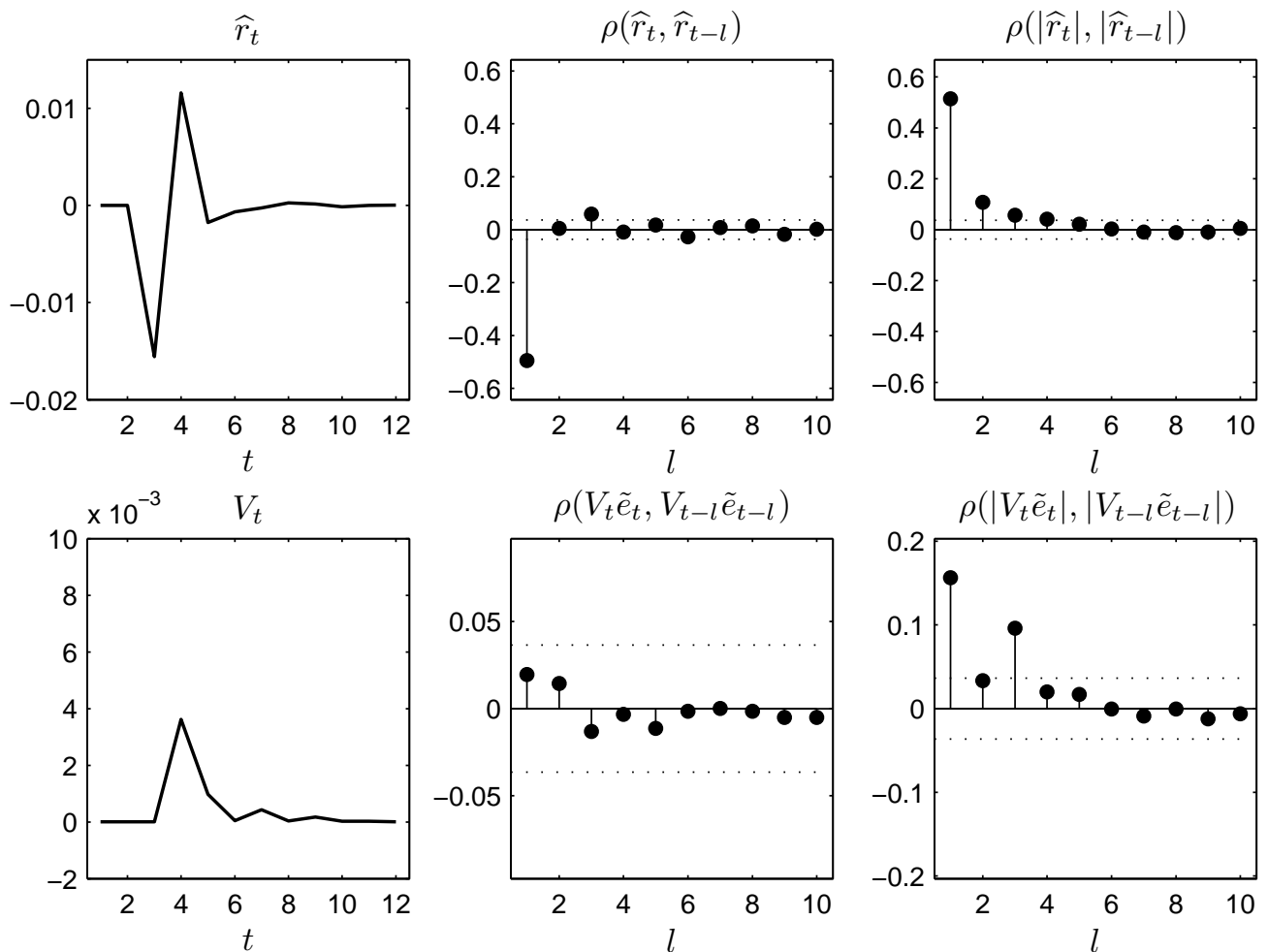

Model with $H=5$, where initial wealth is distributed according to discretized $\beta(2,2)$ distribution, $e \sim N\left(0.03,0.02^{2}\right), \bar{x}=0.75$. Gaussian shocks with variance $\frac{\bar{x}^{2}}{(1-\bar{x})^{2}} \sigma_{\epsilon}^{2}$ are applied to the $\widetilde{r}_{t}$ series at random dates with frequency $\frac{1}{10 \mathrm{H}}$, i.e. on average every 50 points. Autocorrelations are estimated on a 10000 - periods simulation path. 
Figure 2: Shocks to returns, $\cup$-shape wealth distribution

(a) Model Parameters
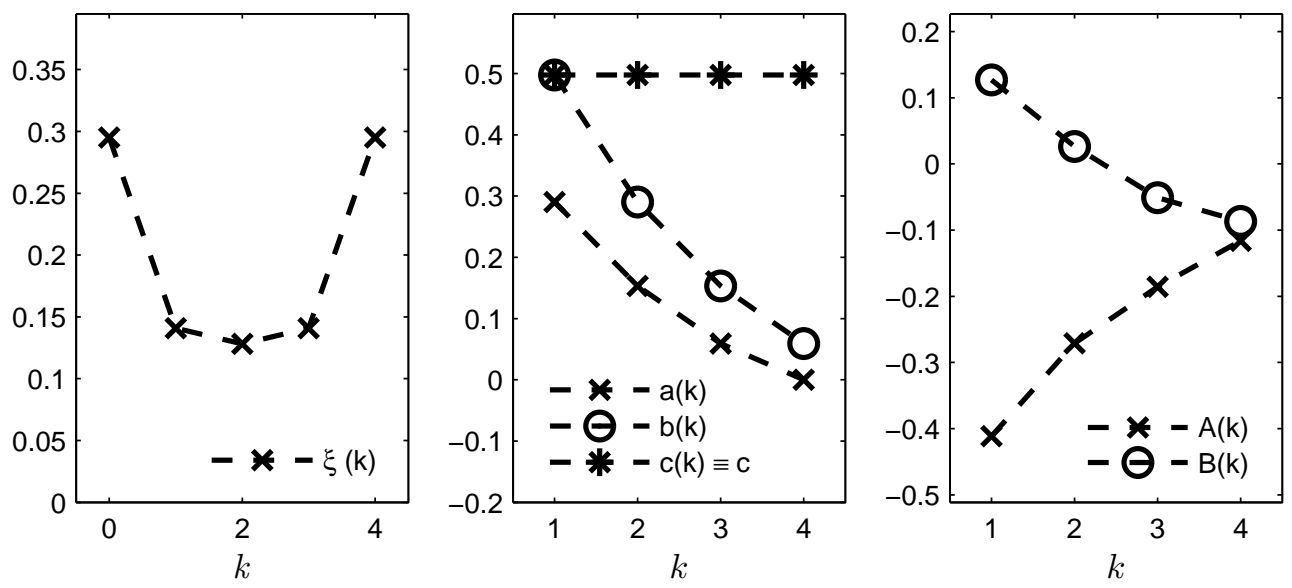

(b) Reaction to Exogenous Shocks
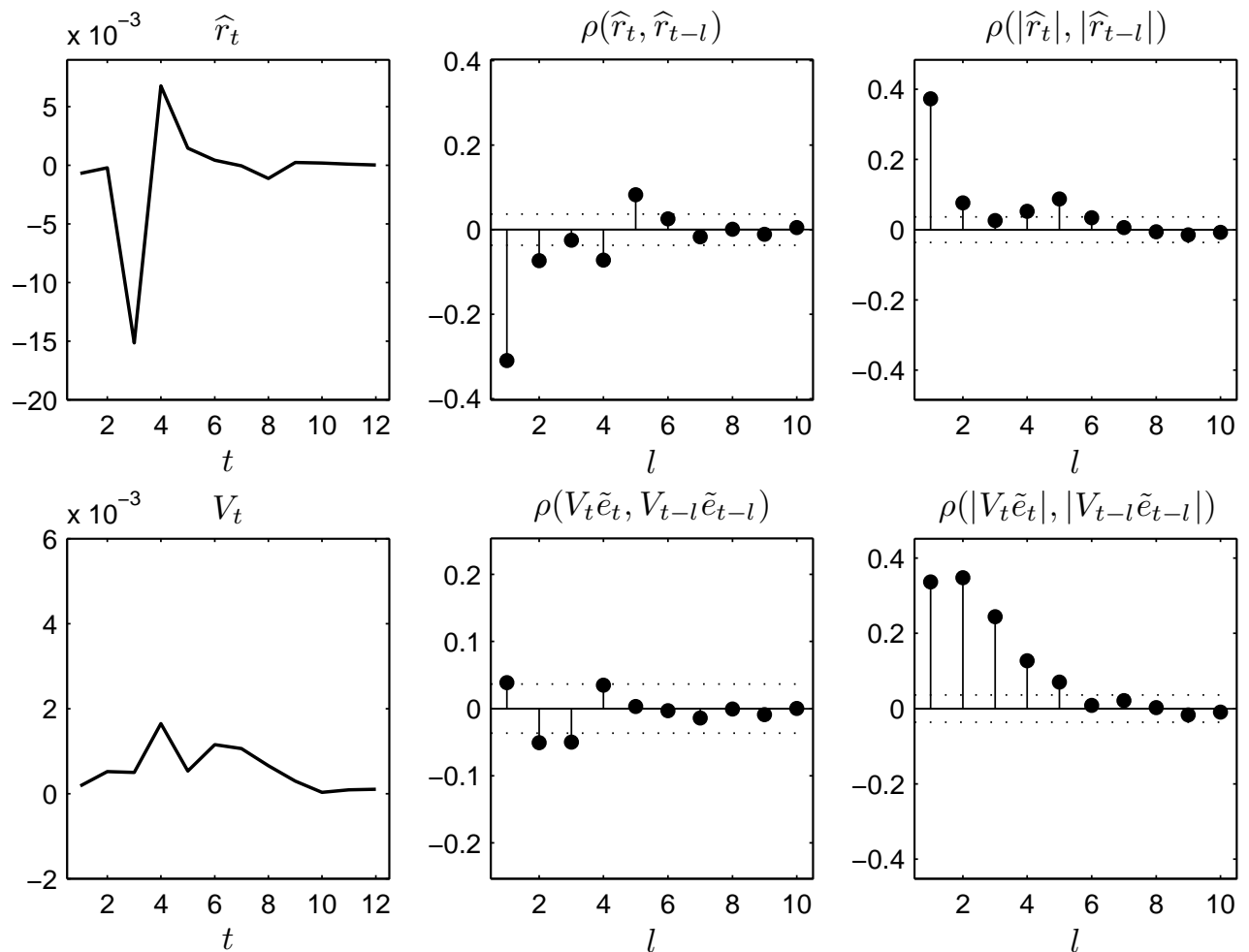

Model with $H=5$, where initial wealth is distributed according to discretized $\beta(2,2)$ distribution, $e \sim N\left(0.03,0.02^{2}\right), \bar{x}=0.75$. Gaussian shocks with variance $\frac{\bar{x}^{2}}{(1-\bar{x})^{2}} \sigma_{\epsilon}^{2}$ are applied to the $\widetilde{r}_{t}$ series at random dates with frequency $\frac{1}{10 H}$, i.e. on average every 50 points. Autocorrelations are estimated on a 10000 - periods simulation path. 
Figure 3: Shocks to returns, wealth shares increase with investment horizon

(a) Model Parameters
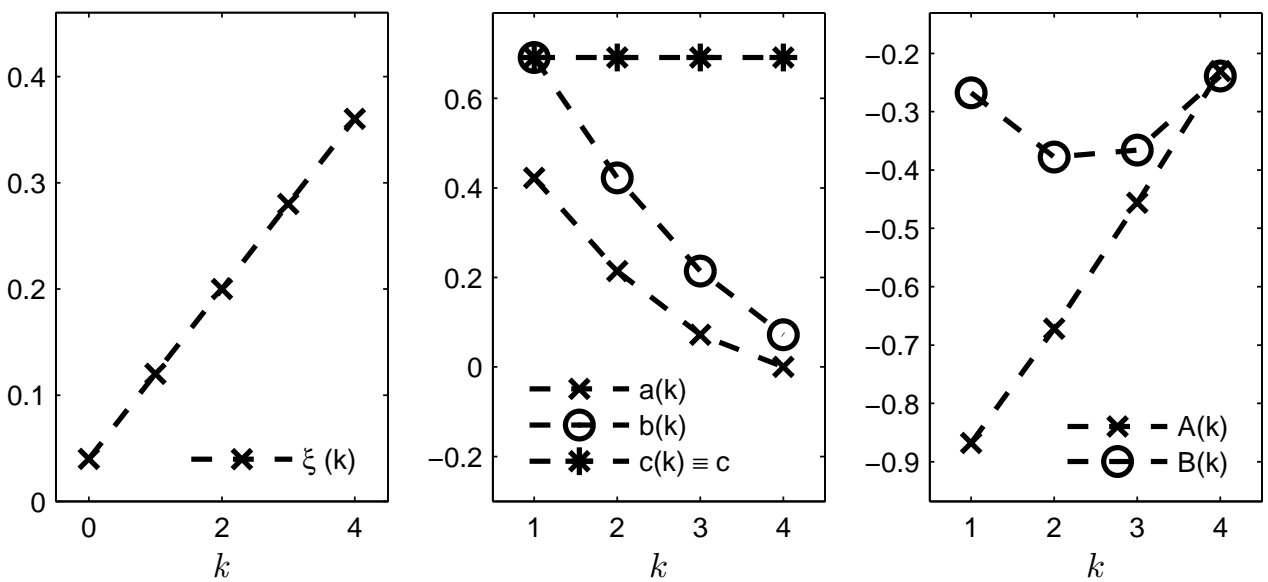

(b) Reaction to Exogenous Shocks
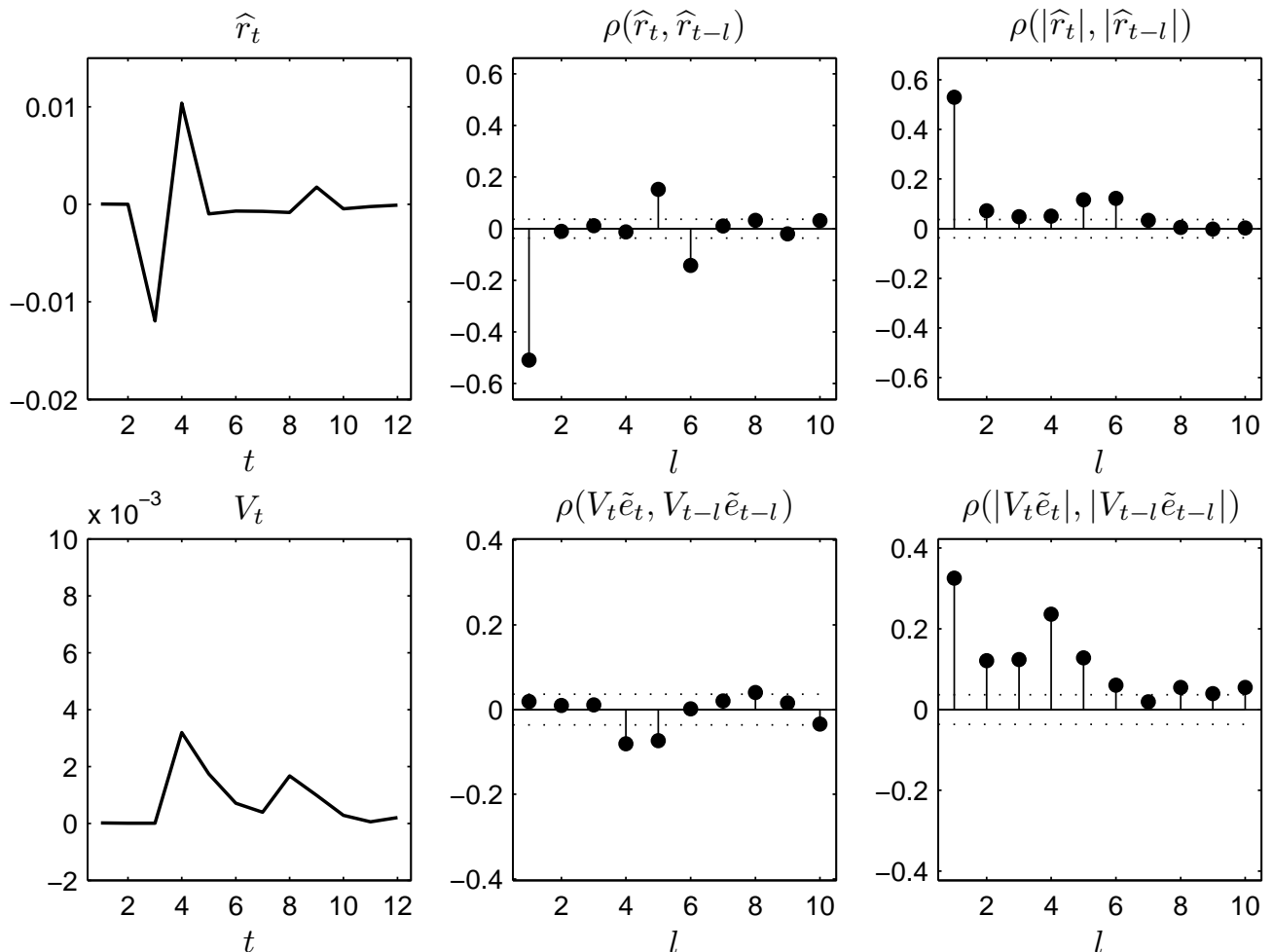

Model with $H=5$, where initial wealth is distributed according to discretized $\beta(2,2)$ distribution, $e \quad N\left(0.03,0.02^{2}\right), \bar{x}=0.75$. Gaussian shocks with variance $\frac{\bar{x}^{2}}{(1-\bar{x})^{2}} \sigma_{\epsilon}^{2}$ are applied to the $\widetilde{r}_{t}$ series at random dates with frequency $\frac{1}{10 \mathrm{H}}$, i.e. on average every 50 points. Autocorrelations are estimated on a 10000 - periods simulation path. 
On Figures 1 - 3 we report the results for three characteristic cases, respectively: $\cap$-shape density of wealth distribution around the maximum at horizon $3, \cup$-shape with symmetric distribution of wealth with maxima at the shortest and longest horizons and the case, when the wealth share linearly increases with investment horizon, so that more wealth is allocated to long-term investors. The first row of plots on each figure represents the wealth distribution and coefficients $a_{k}, b_{k}, c, A_{k}$ and $B_{k}$. The second row represents examples of the trajectory of the error correction term $\hat{r}_{t}$ for 10 periods after a shock occurs and sample autocorrelation function for $\hat{r}_{t}$ and $\left|\hat{r}_{t}\right|$, estimated over a 10000 trading periods simulation path. The third row zooms on the volatility component of the error term, $V_{t}$.

Coefficients $A_{k}$ are always negative at their magnitude decreases with the lag at the rate, depending on the form of the initial wealth distribution. In all cases, this leads to significant anticorrelation with lag one. This result is spectacular because it shows that even without any behavioral or other hypotheses about investment functions our multiscale model generates "contrarian returns".

The presence of small, but significant serial anticorrelations in the series of stock returns is one of the stylized facts about stock price dynamics, known since Fama [1965]. In practice, the presence of statistical arbitrage can reduce these autocorrelations, but these possibilities are limited by various transaction costs, so anticorrelations at daily frequencies often remain noticeable [see Jegadeesh and Titman, 1995]. In more recent studies, anticorrelations in returns are evidenced for many "other than US" markets [see Lee et al., 2003]. Our findings add one more possible explanation.

Non-technically, the error-correction effect in our model can be described as follows. At period $t$ abnormally high (or low) return drives upwards (or downwards) passive investors' share of wealth, invested in the risky asset. At period $t+1$ those of them, who participate in the market, readjust their portfolios to achieve the target allocation. This triggers risky asset return and wealth of passive investors in the direction, opposite to the initial shock. At the next period, investors, who were passive in the previous two periods and currently participate in the trade, readjust their wealth shares with regards to the composite effect of the two previous fluctuations, an so on.

The conditional heteroscedasticity effect is also present and its importance depends on the wealth distribution across scales, with more wealth allocated to longer scales meaning more memory in volatility. However, this effect is relatively small in magnitude and is only slightly reflected in the autocorrelogram of the $\left|\hat{r}_{t}\right|$ series, dominated by the anticorrelation effect. It is almost unnoticeable in the $r_{t}$ series, to which white noise with variance $\frac{\bar{x}}{1-\bar{x}} \sigma_{e}^{2}$ is added (in our example, the noise standard deviation is $3 \times 10^{-3}$ ).

Now consider another type of shock - deviation of the investment function from the equilibrium level. We simulate models with 1-5 horizons, perturbated by random small and non-persistent fluctuations of $x_{t, h}^{0}$, that occur on average once per $10 h$ periods. In Table 1 we report the mean and standard deviation levels for the models with different number of scales. The average return remaining constant, we observe that the global volatility level drops down as the number of horizons increases. In a multi-scale model exogenous shocks are somewhat diluted, because many of the investors do not participate in the trades when the shock occurs. They are, however, affected by the abnormal return, generated 
at this period.

Table 1: Volatility of returns in the multi-scale model with shocks to the investment function

\begin{tabular}{lccccc}
\hline \hline & 1 scale & 2 scales & 3 scales & 4 scales & 5 scales \\
\hline Mean return & 0.009 & 0.009 & 0.009 & 0.009 & 0.009 \\
Volatility & 0.025 & 0.021 & 0.017 & 0.015 & 0.014 \\
\hline \hline
\end{tabular}

Models with $H=1, \ldots, 5$, where initial wealth is distributed according to discretized $\beta(2,2)$ distribution, $e \sim N\left(0.03,0.02^{2}\right), \bar{x}=0.75$. Gaussian shocks with variance $0.01^{2}$ are applied to the demand functions series at random dates with frequency $\frac{1}{10 H}$, i.e. on average every $10-50$ points, depending on investment scale. Parameters are estimated on a 10000 - periods simulation path.

On Figure 4 we represent the dynamic properties of returns in the models with different number of scales. For the one-scale case we observe large abnormal returns at the periods of shocks and large returns at the following period, explained by the reversion to normality of the risky asset weights in investors' portfolios. On the ACF for returns we find strong negative autocorrelation at the first lag, characteristic to the MA(1) process. Note that this is not the case for the shocks in returns, unrelated to demand, that were discussed previously. In the one-scale model, the latter do not trigger subsequent correction.

In the multi-horizon model with $h$ scales, the shocks to $x_{t, h}^{0}$ have impact on the aggregated investment function $x_{t, h}$ during $h-1$ periods, the time necessary to rebalance all portfolios after a shock. This causes a lasting impact on the volatility term $\frac{x_{t}}{1-x_{t}}$ and creates deviations from the equilibrium trajectory, defined by theorem 4.5 with $\hat{r}_{t}=0$. The deviation, in its turn, "activates" the error correction mechanism, described above in details. We find that, in the case of shocks to the demand function, the conditional heteroscedasticity effect is no longer negligible. It manifests itself by the emergence of the significant autocorrelations in absolute returns up to $h-1$ lags. For illustration, we calibrate an MA(1) - GARCH(1,1) model of the form $r_{t}=\mu+\varepsilon_{t}+\alpha \varepsilon_{t-1}, \varepsilon_{t} \sim T\left(0, \sigma_{t}, k\right)$, $\sigma_{t}=c+\phi r_{t-1}+\theta \sigma_{t-1}$ (see Table 2$)$ and find significant moving average and autoregressive components in the conditional volatility process.

For the moment we assumed that disturbances to the system were purely exogenous. We did not suppose any particular type of behavior, such as trendfollowing or contrarian, and we did not make the shocks depend on the past history of returns. It is important that even in this simple case we find that the MIS model can generate interesting dynamic patterns in returns. Certainly, deviations from rational equilibrium in investment functions can be associated to investors' behavior and can be present at every period, unlike our stylized example, designed for illustration purposes.

On Figure 5 we present the results of simulation of a five-scale model with procedurally rational investors that have investment functions, corresponding to definition 3.1 and the equation of the mean expected returns is of the form (11B). For simplicity, we suppose that $L=\infty$, so that the "long term" estimate of average return is the rational equilibrium mean return. We choose $l$ to be equal to $h$, so that the short-term estimate is in fact the last return, observed 
Table 2: Estimation of $\operatorname{GARCH}(1,1)$ in a 5 -scale model

\begin{tabular}{lllr}
\hline \hline & Value & $\begin{array}{c}\text { Standard } \\
\text { Error }\end{array}$ & T-Statistic \\
\hline$\widehat{\mu}$ & 0.0091 & $6 \times 10^{-5}$ & 165.0157 \\
$\widehat{\alpha}$ & -0.4103 & 0.0102 & -40.1992 \\
$\widehat{c}$ & $6 \times 10^{-5}$ & $4 \times 10^{-6}$ & 14.7496 \\
$\widehat{\theta}$ & 0.2684 & 0.0314 & 8.5379 \\
$\widehat{\phi}$ & 0.3702 & 0.0290 & 12.7593 \\
$\widehat{k}$ & 4.2249 & 0.2228 & 18.9672 \\
& & & \\
Log-likelihood: & 31025 & \\
\hline
\end{tabular}

Data generating process: models with 5 horizons, where initial wealth is distributed according to discretized $\beta(2,2)$ distribution, $e \quad N\left(0.03,0.02^{2}\right), \bar{x}=0.75$. Gaussian shocks with variance $0.01^{2}$ are applied to the demand functions series at random dates with frequency $\frac{1}{10 H}$, i.e. on average every $10-50$ points, depending on the investment scale. 10000 simulations. Estimated model specification: $r_{t}=\varepsilon_{t}+\alpha \varepsilon_{t-1}, \varepsilon_{t} \quad T\left(0, \sigma_{t}, k\right), \sigma_{t}=c+\phi r_{t-1}+\theta \sigma_{t-1}$.

on each scale. The parameter $d$, if the behavior of investor is contrarian $(d<0)$ or trend-following $(d>0)$. Note that $d>0$ can also correspond to the case, when investors are fundamentalists, but estimate expected returns on a short historical sample.

Also for simplicity, we assume that agents' strategies are switching, so that the coefficient $d$ can take three values: $d_{T F}=3 \times 10^{-3} \sqrt{h}, d_{C}=1.5 \times 10^{-3} \sqrt{h}$ and $d_{R}=0$. The value $d_{T F}$ is chosen higher than $d_{C}$ because the trend-following behavior seems to be more common, but also because this specification ensures insignificant autocorrellations of returns, making the simulation results more realistic. The normalization factor $\sqrt{h}$ corresponds to the speed of convergence of the empirical mean estimate to the true value and ensures that the magnitude of noise in investment function is the same at all scales. The strategies are chosen independently by investors at each scale according to a Markovian transition matrix. At each period the probability to continue using the same strategy, as in the previous periods, is $1-\frac{1}{10 h}$ and the probability to switch to one of the other two states is $\frac{1}{20 h} 6$.

The resulting dynamics is characterized by insignificant autocorrelations in returns and low, but lasting and significant autocorrelations in absolute returns, which is very characteristic of real stock markets (see, for example Subbotin et al. [2010] or Cont [2001]).

\footnotetext{
${ }^{6}$ We could make the switching endogenous and depending on the success of the corresponding strategy in the past, as in Brock and Hommes [1998]. This would be theoretically more justified but more complicated, while the effect on the return dynamics is barely the same. Our focus being on the multiple scales and not on the behaviorist patterns, we prefer the simpler option.
} 
Figure 4: Shocks to the investment functions
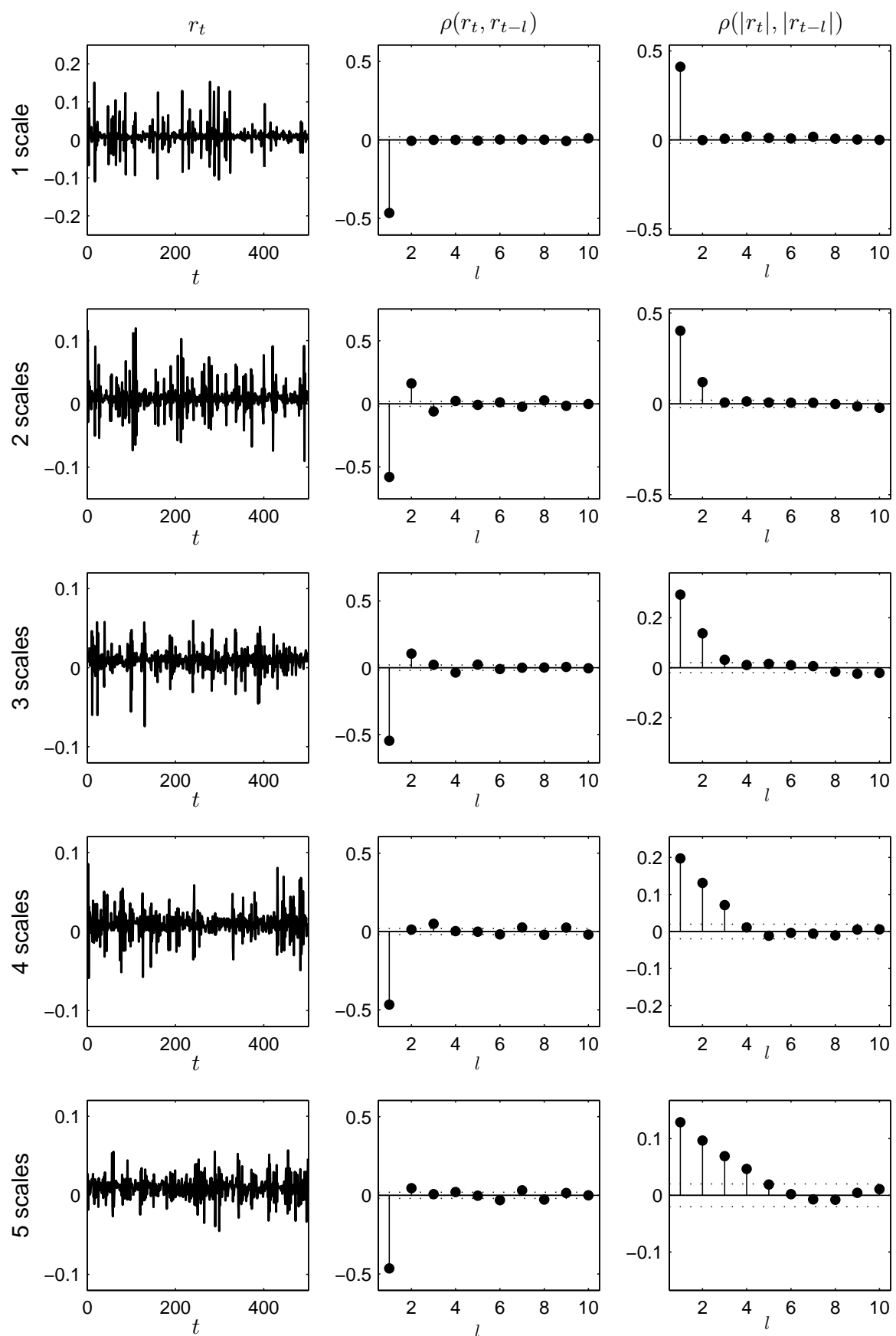

Models with $H=1, \ldots, 5$, where initial wealth is distributed according to discretized $\beta(2,2)$ distribution, $e \approx N\left(0.03,0.02^{2}\right), \bar{x}=0.75$. Gaussian shocks with variance $0.01^{2}$ are applied to the demand functions series at random dates with frequency $\frac{1}{10 H}$, i.e. on average every $10-50$ points, depending on the investment scale. Autocorrelations are estimated on a 10000 - periods simulation path. 
Figure 5: Multiscale model with switching between contrarian and trendfollowing investment
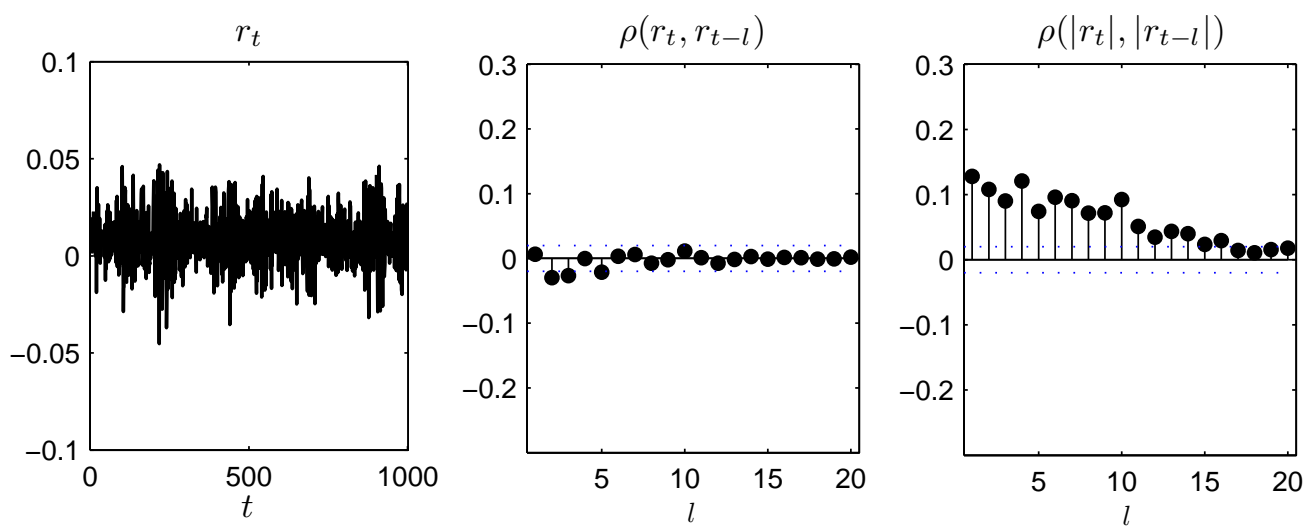

Model with $H=5$, where initial wealth is distributed according to $\beta(2,2)$-distribution, $e \sim$ $N\left(0.03,0.02^{2}\right), \bar{x}=0.75$. Switching rules are described in the text. 10000 simulations.

Figure 6: One-scale model with switching between contrarian and trendfollowing investment

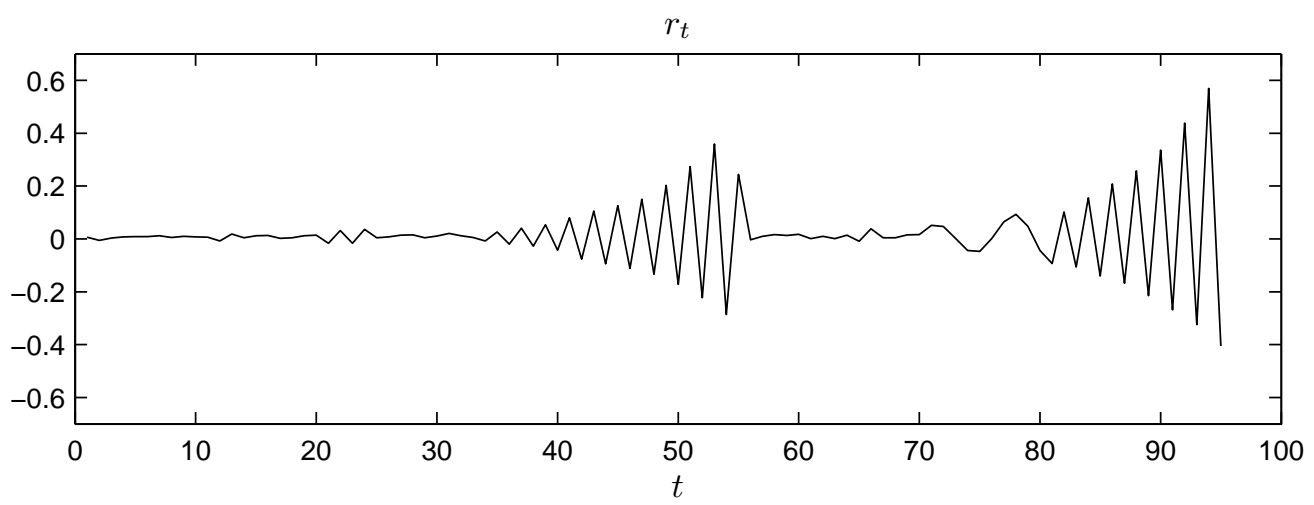

Model with $H=1, e \sim N\left(0.03,0.02^{2}\right), \bar{x}=0.75$. Switching rules are described in the text. Simulation until the market "explodes" (no market clearing possible) - 96 periods. 
Recall that the ARCH-effect can also be obtained in a one-scale model with the same type of investment strategies switching. The important feature of the multi-scale case is that is smooths and mutually mitigates the effects that are present on different scales, so that important deviations from the rational behavior do not lead to explosions of the risky asset price. For illustration, consider the one-scale model with the same investment function, as in our fivescales example. The system is unstable and explodes, as shown on Figure 6 . Thus, introducing multiple scales we extend the set of available strategies and add stability to the dynamic system.

\section{Conclusion}

We have shown that the risky return process in the rational equilibrium for investors with constant relative risk aversion is similar for the case of one and multiple investment scales. However, this result does not hold if the system is subject to shocks, exogenous or related to deviations from rational behavior. In fact, the main difference between the multi-scale and the one-scale models is the way the resulting dynamic system reacts to shocks.

We first demonstrated that the multi-scale model with exogenous shocks to returns displays serial anticorrelations, which is in line with empirical evidence on the so-called contrarian profits. Popular explanations of reversion in the stock returns include overreaction to firm specific information [Jegadeesh and Titman, 1995], measurement errors, related to the bid-ask spread [Boudoukh et al., 1994, Conrad et al., 1997], lead-lag effect, supposing that some stocks react to news faster than others [Lo and MacKinlay, 1990, Chou et al., 2007], time varying systematic risk [Chan, 1988] and some others. Our model contains no overreaction, but includes error correction that consists in temporarily passive market participants' tendency to adjust their portfolios back to the target weights, after the latter have deviated because of price fluctuations. Our theoretical findings contribute to the mean reversion literature, offering one more plausible explanation for the anticorrelation in returns.

More importantly, we demonstrate that the multi-scale model with shocks to investment functions generates conditional heteroscedasticity. Up to now, explanations of conditional heteroscedasticity in the theoretical literature were almost exclusively based on the switching between contrarian and trend-following strategies [Brock and Hommes, 1998, Chiarella and He, 2001, Anufriev et al., 2006, Weinbaum, 2009] or on wealth-dependent relative risk aversion [Vanden, 2005] . Unlike the first class of models, we do not need to stipulate any special patterns in investment behavior. Even exogenous iid disturbances to demand functions generate the GARCH effects. The mechanics of this effect is methodologically close to the relative risk aversion step function model in Vanden [2005]. In both models volatility depends on the demand for the risky asset, expressed as portion of wealth. In Vanden [2005] volatility changes due to the step-wise dependence of the relative risk aversion on wealth. Thus the relation between the price of the risky asset and the value of investment in it cannot remain constant. In our model the demand does not perfectly follow price fluctuations, because some of the agents remain passive at each trading date.

Our multi-scale framework is perfectly compatible with the analysis of the contrarian and the trend-following behavior. Our analysis is restricted to the 
so-called procedurally rational investment strategies, ensuring that, in the case of the iid returns, market participants do not make systematic errors in estimating mean and variance. We prove that in the one-scale case, which was earlier studied in Anufriev et al. [2006], equilibrium returns are never iid, unless procedurally rational investment functions degenerate to the truly rational (constant) investment functions. Naturally, this result also holds for the multi-scale case. One of the consequences of introducing the multiple scales is that the behavioral shocks to investment functions are smoothed in time and lessened in magnitude, which adds stability to the system.

We notify the reader that, though our results are rather general, they are nevertheless based on a series of strong assumptions. The most important of them include $(i)$ the absence of information signals, related to future returns, other than contained in historical prices; (ii) constant and exogenous frequencies of market participants' interventions in the market; (iii) the assumption that the dividend yield is and iid random variable. These assumptions, whose implications on our model are briefly discussed below, can be subjects of further model extensions.

Introducing signals in our model would lead to further heterogeneity of market agents, that will have different times of reaction to news. Obviously, investors acting at large scales would react to news with some delay, which can reinforce the effects, already observed in the multi-scale model. Technically, the model with signals can be close to the one studied in this paper. Our dynamic system is innovated with one variable interpreted as dividend yield. It could be replaced by agents' expectation of expected futures dividends, with one noteworthy difference that this expectation would not be immediately accounted in wealth.

We supposed that frequencies of trading are fixed and exogenous, but they could be made dependent on market conditions. It would be natural to assume that, in a high volatility market, investors tend to readjust their portfolios more often. Trading frequency could be made completely endogenous by introducing implicit transaction costs. In particular, this approach could be helpful in explaining the patterns in trading volume.

Finally, our model, similarly to Chiarella and He [2001] and Anufriev et al. [2006], suggests that the average dividend yield is proportional to price. In economic terms, this means that corporate profits exhibit constant returns to scale, which implies that the equilibrium return can be determined endogenously by the risk-return preferences of the market agents. It would be interesting to study the impact of heterogeneity in the investment behavior in a more consistent macroeconomic setting, where dividend yields are limited by the real economies' productivity.

All the three extensions can be based on the dynamic model with multiple investment scales, studied in this paper. The importance of the results, presented here, is that they establish a general framework, which can be used for further research on more specific problems.

\section{References}

T. Andersen. Return volatility and trading volume: An information flow interpretation of stochastic volatility. Journal of Finance, 51(1):169-204, 1996. 
M. Anufriev. Wealth-Driven Competition in a Speculative Financial Market: Examples with Maximizing Agents. Quantitative Finance, 8(4):363-380, 2008.

M. Anufriev and G. Bottazzi. Asset pricing model with heterogeneous investment horizons. Laboratory of Economics and Management Working Paper Series, SantAnna School for Advanced Studies, 2004.

M. Anufriev, G. Bottazzi, and F. Pancotto. Equilibria, stability and ssymptotic dominance in a speculative market with heterogeneous traders. Journal of Economic Dynamics and Control, 30(9-10):1787-1835, 2006.

N. Barberis and A. Shleifer. Style investing. Journal of Financial Economics, 68(2):161-199, 2003.

J. Boudoukh, M.P. Richardson, and R.F. Whitelaw. A tale of three schools: Insights on autocorrelations of short-horizon stock returns. Review of Financial Studies, 7:539-573, 1994.

W.A. Brock and C.H. Hommes. Heterogeneous beliefs and routes to chaos in a simple asset pricing model. Journal of Economic Dynamics and Control, 22 (8-9):1235-1274, 1998.

K. C. Chan. On the contrarian investment strategy. Journal of Business, 61(2): 147-163, 1988.

T. Chauveau and R. Topol. A unifying microstructure framework for modeling intraday and interday asset pricing dynamics: the case of exchange rates. European Financial Management, 5(3):341-368, 2002.

C. Chiarella and X.-Z. He. Asset price and wealth dynamics under heterogeneous expectations. Quantitative Finance, 1(5):509 - 526, 2001.

P.-H. Chou, K.C. John Wei, and H. Chung. Sources of contrarian profits in the japanese stock market. Journal of Empirical Finance, 14(3):261-286, 2007.

J. Conrad, M. Gultekin, and G. Kaul. Profitability of short-term contrarian strategies: Implications for market efficiency. Journal of Business $\&$ Economic Statistics, 15(3):379-386, 1997.

R. Cont. Empirical properties of asset returns: Stylized facts and statistical issues. Quantitative Finance, 1(2):223-236, 2001.

E. Fama. The behaviour of stock market prices. Journal of Business, 38:34-105, 1965.

C. Hommes. Heterogeneous agent models in economics and finance. In Handbook of Computational Economics, volume 2, pages 1109-1186, Amsterdam, 2006. Elsevier Science.

N. Jegadeesh and S. Titman. Overreaction, delayed reaction, and contrarian profits. Review of Financial Studies, 8(4):973993, 1995.

B. LeBaron. Agent-based computational finance. In Handbook of Computational Economics, volume 2, pages 1187-1233, Amsterdam, 2006. Elsevier Science. 
D. Lee, H. Chan, R. Faff, and P. Kalev. Short-term contrarian investing is it profitable?... yes and no. Journal of Multinational Financial Management, 13(4-5):385-404, 2003.

A. Lo and C. MacKinlay. When are contrarian profits due to stock market overreaction? Review of Financial Studies, 3:175-250, 1990.

U. Müller, M. Dacorogna, R. Dave, R. Olsen, O. Pictet, and J. Von Weizsacker. Volatilities of different time resolutions - analyzing the dynamics of market components. Journal of Empirical Finance, 4:213-239, 1997.

B. Ninness. Strong laws of large numbers under weak assumptions with application. IEEE Transactions on Automatic Control, 45(11):2117-2122, 2000.

H. Simon. From substantive to procedural rationality. In S. J. Latsis, editor, Method and Appraisal in Economics, pages 129-148. Cambridge University Press, 1976.

A. Subbotin, T. Chauveau, and K. Shapovalova. Volatility models: from garch to multi-horizon cascades. In B. Naas and J. Lysne, editors, Financial Markets and the Global Recession. Nova Science Publishers Inc, NY (forthcoming), 2010 .

J. Vanden. Equilibrium analysis of volatility clustering. Journal of Empirical Finance, 12:374-417, 2005.

D. Weinbaum. Investor heterogeneity, asset pricing and volatility dynamics. Journal of Economic Dynamics and Control, 33:1379-1397, 2009. 


\section{Appendix}

\section{Proof of proposition 2.2}

Proof. Consider an investor, who does not operate on the market in current period. He has a portion $x_{t}$ of his wealth invested in the risky asset. In the next period the wealth he detains in the risky shares becomes (we omit the subscript $i$ for simplicity):

$$
\frac{x_{t} W_{t} P_{t+1}}{P_{t}}=\frac{x_{t} w_{t} p_{t+1}\left(1+R_{f}\right)^{t+1}}{p_{t}}=x_{t} w_{t}\left(1+r_{t+1}\right)\left(1+R_{f}\right)^{t+1}
$$

His wealth invested in the risk-free asset reads:

$$
\begin{gathered}
W_{t}\left(1-x_{t}\right)\left(1+R_{f}\right)+\frac{D_{t+1} W_{t} x_{t}}{P_{t}}=w_{t}\left(1-x_{t}\right)\left(1+R_{f}\right)^{t+1}+ \\
+e_{t+1} w_{t} x_{t}\left(1+R_{f}\right)^{t+1}=\left(1+R_{f}\right)^{t+1} w_{t}\left[1-x_{t}\left(1-e_{t+1}\right)\right]
\end{gathered}
$$

Therefore, the share of investment in the risky asset satisfies:

$$
\begin{gathered}
x_{t+1}^{-1}=\frac{x_{t} w_{t}\left(1+r_{t+1}\right)\left(1+R_{f}\right)^{t+1}}{x_{t} w_{t}\left(1+r_{t+1}\right)\left(1+R_{f}\right)^{t+1}+\left(1+R_{f}\right)^{t+1} w_{t}\left[1-x_{t}\left(1-e_{t+1}\right)\right]}= \\
=\frac{x_{t}\left(1+r_{t+1}\right)}{1+x_{t}\left(r_{t+1}+e_{t+1}\right)}
\end{gathered}
$$

Equation (4) is obtained if instead of $x_{t}$ the same argument is applied to $x_{t-1}^{-k+1}$.

\section{Proof of Proposition 2.3}

Proof. The evolution of an investor's wealth between $t$ and $t+h$ reads (we drop the subscript $i$ to ease the notation):

$$
W_{t+h}=x_{t} \frac{W_{t}}{P_{t}}\left(P_{t+h}+\sum_{k=1}^{h} D_{t+k}\left(1+R_{f}\right)^{h-k}\right)+\left(1-x_{t}\right) W_{t}\left(1+R_{f}\right)^{h} .
$$

Let $Y_{t, t+h}$ be the total return on the risky asset for the passive investor between $t$ and $t+h$. Then we have:

$$
\begin{gathered}
Y_{t, t+h}=\frac{P_{t+h}}{P_{t}}+\sum_{k=1}^{h} \frac{D_{t+k}}{P_{t}}\left(1+R_{f}\right)^{h-k}-1= \\
\prod_{k=1}^{h}\left(1+R_{t+k}\right)+\sum_{k=1}^{h} \varepsilon_{t+k} \prod_{i=1}^{k-1}\left(1+R_{t+i}\right)\left(1+R_{f}\right)^{h-k}-1 .
\end{gathered}
$$

The solution of the maximization problem (6) reads:

$$
x_{t}^{*}=\frac{1+\mathrm{E}_{t-1}\left[Y_{t, t+h}\right]-\left(1+R_{f}\right)^{h}}{\gamma \operatorname{Var}_{t-1}\left[Y_{t, t+h}\right]} .
$$

For small returns, (A-1) can be approximated by:

$$
x_{t, i}^{*}=\frac{1+\mathrm{E}_{t-1}\left[Y_{t, t+h}\right]-h R_{f}}{\gamma_{i} \operatorname{Var}_{t-1}\left[Y_{t, t+h}\right]},
$$


so that in terms of rescaled variables the total riksy return can be written:

$$
\begin{gathered}
1+Y_{t, t+h} \stackrel{\text { def }}{=}\left(1+R_{f}\right)^{h}\left(1+y_{t, t+h}\right)= \\
\left(1+R_{f}\right)^{h}\left[\prod_{k=1}^{h}\left(1+r_{t+k}\right)+\sum_{k=1}^{h} e_{t+k} \prod_{i=1}^{k-1}\left(1+r_{t+i}\right)\right],
\end{gathered}
$$

or with the first order precision:

$$
1+Y_{t, t+h} \approx 1+y_{t, t+h} \approx h R_{f}+\sum_{k=1}^{h} r_{t+k}+e_{t+k} .
$$

For the optimal investment share, this gives approximative solution:

$$
x_{t, i}^{*} \approx \frac{\mathrm{E}_{t-1}\left[y_{t, t+h}\right]}{\gamma_{i} \operatorname{Var}_{t-1}\left[y_{t, t+h}\right]}
$$

\section{Proof of Theorem 3.3}

Proof. Equation (12) can be written in the form:

$$
r_{t}=\frac{x_{t}-x_{t-1}+\bar{e} x_{t} x_{t-1}}{x_{t-1}-x_{t} x_{t-1}}+\frac{x_{t} x_{t-1}}{x_{t-1}-x_{t} x_{t-1}} \widetilde{e}_{t} .
$$

We define the function:

$$
F\left(r_{t-1}, \ldots, r_{t-L-1}\right)=\frac{x_{t}-x_{t-1}+\bar{e} x_{t} x_{t-1}}{x_{t-1}-x_{t} x_{t-1}} .
$$

The function $F$ depends on the history of returns upto the lag $L+1$ because it the term $x_{t-1}$, determined by investors from $L$ observations of returns prior to $t-1$. Denote $F_{k}^{\prime}$ the derivative of $F$ with respect to its argument number $k$, evaluated in the point $(\bar{r}, \ldots, \bar{r})$. Then we have:

$$
\begin{gathered}
F_{1}^{\prime}=\frac{\left(f_{1}^{\prime}+f_{1}^{\prime} \bar{x} \bar{e}\right)[\bar{x}(1-\bar{x})]+\bar{x} f_{1}^{\prime}\left(\bar{x}-\bar{x}+\bar{x}^{2} \bar{e}\right)}{\bar{x}^{2}(1-\bar{x})^{2}}= \\
\frac{f_{1}^{\prime}[1-\bar{x}(1-\bar{e})]}{\bar{x}(1-\bar{x})^{2}}
\end{gathered}
$$

For $k=2, \ldots, L$ we obtain:

$$
\begin{gathered}
F_{k}^{\prime}=\frac{\bar{x}(1-\bar{x})\left[f_{k}^{\prime}-f_{k-1}^{\prime}+\bar{x} \bar{e}\left(f_{k}^{\prime}+f_{k-1}^{\prime}\right)\right]-\bar{x}^{2} \bar{e}\left[f_{k-1}^{\prime}(1-\bar{x})-\bar{x} f_{k}^{\prime}\right]}{\bar{x}^{2}(1-\bar{x})^{2}}= \\
\frac{f_{k}^{\prime}[1-\bar{x}(1-\bar{e})]+f_{k-1}^{\prime}(\bar{x}-1)}{\bar{x}(1-\bar{x})^{2}}
\end{gathered}
$$

Finally, for the last term:

$$
F_{L+1}^{\prime}=\frac{\bar{x}(1-\bar{x})\left(-f_{L}^{\prime}+\bar{x} \bar{e} f_{L}^{\prime}\right)-\bar{x}^{2} \bar{e}(1-\bar{x}) f_{L}^{\prime}}{\bar{x}^{2}(1-\bar{x})^{2}}=-\frac{f_{L}^{\prime}}{\bar{x}(1-\bar{x})}
$$


In a similar way, define

$$
G\left(r_{t-1}, \ldots, r_{t-k-1}\right)=\frac{x_{t} x_{t-1}}{x_{t-1}-x_{t} x_{t-1}}
$$

and compute its derivatives:

$$
G_{1}^{\prime}=\frac{\bar{x}(1-\bar{x}) \bar{x} f_{1}^{\prime}+\bar{x} f_{1}^{\prime} \bar{x}^{2}}{\bar{x}^{2}(1-\bar{x})^{2}}=\frac{f_{1}^{\prime}}{(1-\bar{x})^{2}}
$$

For $k=2, \ldots, L$ :

$$
G_{k}^{\prime}=\frac{\left[\bar{x} f_{k}^{\prime}+\bar{x} f_{k-1}^{\prime}\right] \bar{x}(1-\bar{x})-\bar{x}^{2}\left[f_{k-1}^{\prime}(1-\bar{x})-\bar{x} f_{k}^{\prime}\right]}{\bar{x}^{2}(1-\bar{x})^{2}}=\frac{f_{k}^{\prime}}{(1-\bar{x})^{2}}
$$

The last term reads:

$$
G_{L+1}^{\prime}=\frac{\bar{x} f_{L}^{\prime} \bar{x}(1-\bar{x})-\bar{x}^{2} f_{L}^{\prime}(1-b x)}{\bar{x}^{2}(1-\bar{x})^{2}}=0
$$

Using these results, we can rewrite the stochastic process (12) for returns in a linearized form:

$$
\begin{gathered}
\widetilde{r}_{t}=-\bar{r}+F(\bar{r}, \ldots, \bar{r})+\sum_{k=1}^{L+1} F_{i}^{\prime} \widetilde{r}_{t-k}+v_{t} \widetilde{e}_{t} \\
v_{t}=G(\bar{r}, \ldots, \bar{r})+\sum_{k=1}^{L} G_{i}^{\prime} \widetilde{r}_{t-k}
\end{gathered}
$$

Since $\widetilde{r}_{t}$ is a zero-mean process, we impose:

$$
\bar{r}=F(\bar{r}, \ldots, \bar{r})=\frac{\bar{x} \bar{e}}{1-\bar{x}}
$$

which immediately gives (19).

\section{Proof of Theorem 3.4}

Proof. The equilibrium dynamics of returns can be derived from (2) in a way, similar to the homogeneous case:

$$
r_{t}=\frac{\sum_{i} w_{t-1, i}\left(x_{t, i}-x_{t-1, i}\right)+e_{t} \sum_{i} x_{t, i} x_{t-1, i} w_{t-1, i}}{\sum_{i} w_{t-1, i}\left(x_{t-1, i}-x_{t, i} x_{t-1, i}\right)}
$$

If $r_{t}$ is $i i d$, the same is true for $r_{t \mid t-1}$. For the mean of $r_{t \mid t-1}$ to be constant, it is necessary that:

$$
\begin{gathered}
\sum_{i} \phi_{t-1, i}\left(x_{t, i}-x_{t-1, i}\right)=c_{1} \sum_{i} \phi_{t-1, i}\left(x_{t-1, i}-x_{t, i} x_{t-1, i}\right) \\
\sum_{i} \phi_{t-1, i} x_{t, i} x_{t-1, i}=\tilde{c}_{2}\left(x_{t-1, i}-x_{t, i} x_{t-1, i}\right)
\end{gathered}
$$


with $c_{1}$ and $\tilde{c}_{2}$ two constants and $\phi_{t, i}=\frac{w_{t, i}}{\sum_{i} w_{t, i}}$. Then $\mathrm{E}_{\mathrm{t}-1}\left(r_{t}\right)=c_{1}+\tilde{c}_{2} \bar{e}$ and $\operatorname{Var}_{\mathrm{t}-1}\left(r_{t}\right)=\tilde{c}_{2}^{2} \sigma_{e}^{2}$. Simplifying (A-3) and setting $c_{2}=\frac{\tilde{c}_{2}}{1+\tilde{c}_{2}}$, we get:

$$
\begin{gathered}
\sum_{i} \phi_{t-1, i} x_{t, i}=\left[1-c_{1}\left(1-c_{2}\right)\right] \sum_{i} \phi_{t-1, i} x_{t-1, i} \\
\sum_{i} \phi_{t-1, i} x_{t, i} x_{t-1, i}=c_{2} \sum_{i} \phi_{t-1, i} x_{t-1, i}
\end{gathered}
$$

with $c_{1}$ and $c_{2}$ are two constants. The case of constant average investment function is a particular case of $(\mathrm{A}-4)$ when we chose $c_{1}=0$. Note that the quantity $\sum_{i} \phi_{t-1, i} x_{t, i} x_{t-1, i}$ must also be constant in this case.

On the other hand, if returns are iid, procedurally rational investors must have investment functions, based on unbiased beliefs. Thus, for any $t$ the latter can be written in the form:

$$
x_{t, i}=\frac{\mathrm{E}\left(r_{t}\right)+v_{t, i}}{\gamma_{i} \operatorname{Var}\left(r_{t}\right)+\zeta_{t, i}}
$$

with $v_{t, i}$ and $\zeta_{t, i}$ are centered random variables. But (A-5) implies that in (A4) the growth rate of the aggregate investment share $1-c_{1}\left(1-c_{2}\right)$ cnanot be deterministic and different from one. Thus the case of the constant average investment function, where $c_{1}=0$, is the only one compatible with the iid dynamics of returns in the heterogeneous case. The mean and variance of returns in this case are proportional to the mean and variance of the dividend yield, but are not defined uniquely and depend on the parameter $c_{2}$.

\section{Proof of Theorem 4.1}

Proof. Let us denote

$$
\begin{aligned}
\Xi(r) & =r-\frac{\sum_{h=1}^{H} w_{t-1, h}\left(x_{t, h}-x_{t-1, h}\right)+e_{t} \sum_{h=1}^{H} x_{t, h} x_{t-1, h} w_{t-1, h}}{\sum_{h=1}^{H} w_{t-1, h} x_{t-1, h}\left(1-x_{t, h}\right)} \\
x_{t, h} & =\frac{1}{h} \sum_{k=0}^{h-1} x_{t, h}^{-k} \\
x_{t, h}^{-k} & =\frac{x_{t-1, h}^{-k+1}(1+r)}{1+x_{t-1, h}^{-k+1}\left(r+e_{t}\right)}
\end{aligned}
$$

We prove that continuous function $\Xi(r)$ at least once takes the value zero in the interval ] $-1 ; \infty[$ (the lower bound for $r$ ensures that stock price stays positive). It is easy to show that:

$$
\lim _{r \rightarrow-1} \Xi(r)<0
$$

First, notice that $\lim _{r \rightarrow-1} x_{t, h}^{-k}=0, \forall k$, and so the right side of the expression for $\Xi(r)$ does not depend on $r$. Then, using that $0 \leq x_{t, h} \leq 1$ and by consequence

$$
\sum_{h=1}^{H} w_{t-1, h} x_{t-1, h}\left(1-x_{t, h}\right)>0,
$$


we can show that the statement $\lim _{r \rightarrow-1} \Xi(r)>0$ is equivalent to:

$$
-\sum_{h=1}^{H} w_{t-1, h} x_{t-1, h}\left(1-x_{t, h}\right)-\sum_{h=1}^{H} w_{t-1, h}\left(x_{t, h}-x_{t-1, h}\right)-e_{t} \sum_{h=1}^{H} x_{t, h} x_{t-1, h} w_{t-1, h}>0 .
$$

The latter inequality simplifies to:

$$
-\sum_{h=1}^{H} w_{t-1, h} x_{t-1, h} x_{t, h}-\sum_{h=1}^{H} w_{t-1, h} x_{t, h}-\sum_{h=1}^{H} w_{t-1, h} x_{t, h}, x_{t-1, h} e_{t}>0
$$

which is evidently absurd.

On the other hand, one can show that:

$$
\lim _{r \rightarrow \infty} \Xi(r)=\infty
$$

This follows from $\lim _{r \rightarrow \infty} x_{t, h}^{-k}=1, \forall k$, which implies that:

$$
\lim _{r \rightarrow \infty}=\frac{\sum_{h=1}^{H} w_{t-1, h}\left(x_{t, h}-x_{t-1, h}\right)+e_{t} \sum_{h=1}^{H} x_{t, h} x_{t-1, h} w_{t-1, h}}{\sum_{h=1}^{H} w_{t-1, h} x_{t-1, h}\left(1-x_{t, h}\right)}<\infty .
$$

The result of the theorem is obtained by continuity.

\section{Proof of Theorem 4.3}

Proof. Denote $\xi_{t, i, j}$ the ratio between the wealth of investors at scale $i$ and at scale $j$, taken at time $t$, that is:

$$
\xi_{t, i, j}=\frac{w_{t, i}}{w_{t, j}}
$$

It is evident that definition (24) is equivalent to

$$
\xi_{0, i, j}>0=>P\left(\xi_{t, i, j}=0\right)=0, \forall i, j \in\{1, \ldots, H\} \text {, when } t \rightarrow \infty
$$

The ratio of wealth can be written as:

$$
\xi_{t, i, j}=\xi_{0, i, j} \frac{\prod_{k=1}^{t} g_{k, i}}{\prod_{k=1}^{t} g_{k, j}}
$$

or equivalently in logarithmic terms:

$$
\ln \left(\xi_{t, i, j}\right)=\ln \left(\xi_{0, i, j}\right)+\sum_{k=1}^{t}\left[\ln \left(g_{k, i}\right)-\ln \left(g_{k, j}\right)\right] .
$$

It follows from (24) that, as $t \rightarrow \infty$, the sum in (A-7) converges almost surely to:

$$
\sum_{k=1}^{\infty}\left(\mathrm{E}\left[\ln \left(g_{k, i}\right)\right]-\mathrm{E}\left[\ln \left(g_{k, i}\right)\right]\right)
$$

which is finite if and only if

$$
\mathrm{E}\left[\ln \left(g_{k, i}\right)\right]=\mathrm{E}\left[\ln \left(g_{k, i}\right)\right] .
$$

This condition is evidently equivalent to:

$$
\ln \left(\xi_{t, i, j}\right) \stackrel{a . s .}{\longrightarrow} \ln \left(\xi_{0, i, j}\right)
$$

which proves the theorem. 


\section{Proof of Theorem 4.5}

Proof. Consider equation $F\left(r_{1}, \ldots, r_{t-1}, e_{1}, \ldots, e_{t-1}, r_{t}, e_{t}\right)=0$ that implicitly defines the equilibrium return dynamics. In order to study conditional volatility of returns, we will linearize the function $F$ in the neighborhood of the point $M_{\epsilon} \stackrel{\text { def }}{=}\left(\bar{r}, \ldots, \bar{r}, \bar{e}, \ldots, \bar{e}, \bar{r}, e_{t}\right)$ rather than the point $M$ (recall that $e_{t}$ is the source of innovation in the system). A first-order series expansion yields:

$$
F\left(M_{\epsilon}\right)+\sum_{i=1}^{t} \frac{\partial F}{\partial r_{t-k}}\left(M_{\epsilon}\right) \widetilde{r}_{t-k}+\sum_{k=1}^{t} \frac{\partial F}{\partial e_{t-k}}\left(M_{\epsilon}\right) \widetilde{e}_{t-k}+\frac{\partial F}{\partial r_{t}}\left(M_{\epsilon}\right) \widetilde{r}_{t}=0
$$

This implies that the return satisfies:

$$
\widetilde{r}_{t}=-\frac{F\left(M_{\epsilon}\right)}{\frac{\partial F}{\partial r_{t}}\left(M_{\epsilon}\right)}-\sum_{k=1}^{t} \frac{\frac{\partial F}{\partial r_{t-k}}\left(M_{\epsilon}\right)}{\frac{\partial F}{\partial r_{t}}\left(M_{\epsilon}\right)} \widetilde{r}_{t-k}-\sum_{k=1}^{t} \frac{\frac{\partial F}{\partial e_{t-k}}\left(M_{\epsilon}\right)}{\frac{\partial F}{\partial r_{t}}\left(M_{\epsilon}\right)} \widetilde{e}_{t-k} .
$$

The share of investment in the risky asset is a function of returns and dividend yields, given by the last two equations of (23). We denote:

$$
\begin{aligned}
\bar{x} & =x_{t-1, h}\left(M_{\epsilon}\right), \\
x_{h, \epsilon}=x_{t, h}\left(M_{\epsilon}\right) & =\frac{1}{h}\left[\bar{x}+(h-1) \frac{\bar{x}(1+\bar{r})}{1+\bar{x}\left(\bar{r}+e_{t}\right)}\right], \\
x_{\epsilon} & =\sum_{h=1}^{H} \xi_{0, h} x_{h, \epsilon} .
\end{aligned}
$$

Now let us obtain the explicit expressions for all elements of equation (A-9):

$$
F\left(M_{\epsilon}\right)=\frac{x_{\epsilon}-\bar{x}+\bar{x} x_{\epsilon} e_{t}}{\bar{x}\left(1-x_{\epsilon}\right)}-\bar{r}
$$

The partial derivative of $F$ with respect to the past return $r_{t-k}, k=1, \ldots, t$ 
reads:

$$
\begin{aligned}
& \frac{\partial F}{\partial r_{t-k}}\left(M_{\epsilon}\right)=\left\{\left[\sum_{h=1}^{H} \frac{\partial w_{t-1, h}}{\partial r_{t-k}}\left(M_{\epsilon}\right)\left(x_{h, \epsilon}-\bar{x}+\bar{x} x_{h, \epsilon} e_{t}\right)+\right.\right. \\
& \left.\sum_{h=1}^{H} \bar{w}_{t-1, h}\left(\frac{\partial x_{t, h}}{\partial r_{t-k}}\left(M_{\epsilon}\right)-\frac{\partial x_{t-1, h}}{\partial r_{t-k}}\left(M_{\epsilon}\right)+\frac{\partial\left(x_{t-1, h} x_{t, h}\right)}{\partial r_{t-k}}\left(M_{\epsilon}\right) e_{t}\right)\right] \times \\
& {\left[\sum_{h=1}^{H} \bar{w}_{t-1, h} \bar{x}\left(1-x_{h, \epsilon}\right)\right]-\left[\sum_{h=1}^{H} \frac{\partial w_{t-1, h}}{\partial r_{t-k}}\left(M_{\epsilon}\right) \bar{x}\left(1-x_{h, \epsilon}\right)+\right.} \\
& \left.\sum_{h=1}^{H} \bar{w}_{t-1, h}\left(\frac{\partial x_{t-1, h}}{\partial r_{t-k}}\left(M_{\epsilon}\right)-\frac{\partial\left(x_{t-1, h} x_{t, h}\right)}{\partial r_{t-k}}\left(M_{\epsilon}\right)\right)\right] \times \\
& \left.\left[\sum_{h=1}^{H} \bar{w}_{t-1, h}\left(x_{h, \epsilon}-\bar{x}+\bar{x} x_{h, \epsilon} e_{t}\right)\right]\right\} \times\left[\sum_{h=1}^{H} \bar{w}_{t-1, h} \bar{x}\left(1-x_{h, \epsilon}\right)\right]^{-2}= \\
& \left\{\sum_{h=1}^{H} \xi_{0, h}\left(\frac{\partial x_{t, h}}{\partial r_{t-k}}\left(M_{\epsilon}\right)-\frac{\partial x_{t-1, h}}{\partial r_{t-k}}\left(M_{\epsilon}\right)+\frac{\partial\left(x_{t-1, h} x_{t, h}\right)}{\partial r_{t-k}}\left(M_{\epsilon}\right) e_{t}\right) \times\right. \\
& \sum_{h=1}^{H} \xi_{0, h} \bar{x}\left(1-x_{h, \epsilon}\right)-\sum_{h=1}^{H} \xi_{0, h}\left(\frac{\partial x_{t-1, h}}{\partial r_{t-k}}\left(M_{\epsilon}\right)-\frac{\partial\left(x_{t-1, h} x_{t, h}\right)}{\partial r_{t-k}}\left(M_{\epsilon}\right)\right) \times \\
& \left.\sum_{h=1}^{H} \xi_{0, h}\left(x_{h, \epsilon}-\bar{x}+\bar{x} x_{h, \epsilon} e_{t}\right)\right\} \times\left[\sum_{h=1}^{H} \xi_{0, h} \bar{x}\left(1-x_{h, \epsilon}\right)\right]^{-2}= \\
& \frac{1}{\bar{x}^{2}\left(1-x_{\epsilon}\right)^{2}}\left\{\sum _ { h = 1 } ^ { H } \xi _ { 0 , h } \left[\frac{\partial x_{t, h}}{\partial r_{t-k}}\left(M_{\epsilon}\right)\left(\bar{x}\left(1-x_{\epsilon}\right)+\bar{x}\left(\bar{x} e_{t}+x_{\epsilon}-\bar{x}\right)\right)+\right.\right. \\
& \left.\left.\frac{\partial x_{t-1, h}}{\partial r_{t-k}}\left(M_{\epsilon}\right)\left(x_{\epsilon}\left(\bar{x} e_{t}+x_{\epsilon}-\bar{x}\right)-\left(-\bar{x} x_{\epsilon}+x_{\epsilon}+\bar{x} x_{\epsilon} e_{t}\right)\right)\right]\right\}= \\
& \frac{\sum_{h=1}^{H} \xi_{0, h}\left[\frac{\partial x_{t, h}}{\partial r_{t-k}}\left(M_{\epsilon}\right)\left(\bar{x}+\bar{x}^{2} e_{t}-\bar{x}^{2}\right)+\frac{\partial x_{t-1, h}}{\partial r_{t-k}}\left(M_{\epsilon}\right)\left(x_{\epsilon}{ }^{2}-x_{\epsilon}\right)\right]}{\bar{x}^{2}\left(1-x_{\epsilon}\right)^{2}} .
\end{aligned}
$$

The derivative of $F$ with respect to the current return reads:

$$
\frac{\partial F}{\partial r_{t}}\left(M_{\epsilon}\right)=\frac{\sum_{h=1}^{H} \xi_{0, h} \frac{\partial x_{t, h}}{\partial r_{t}}\left(M_{\epsilon}\right)\left(\bar{x}+\bar{x}^{2} e_{t}-\bar{x}^{2}\right)}{\bar{x}^{2}\left(1-x_{\epsilon}\right)^{2}}-1 .
$$

In a similar way, we find the derivative of $F$ with respect to the past dividend yield $e_{t-k}, k=1, \ldots, t$ :

$$
\begin{gathered}
\frac{\partial F}{\partial e_{t-k}}\left(M_{\epsilon}\right)= \\
\frac{\sum_{h=1}^{H} \xi_{0, h}\left[\frac{\partial x_{t, h}}{\partial e_{t-k}}\left(M_{\epsilon}\right)\left(\bar{x}+\bar{x}^{2} e_{t}-\bar{x}^{2}\right)+\frac{\partial x_{t-1, h}}{\partial e_{t-k}}\left(M_{\epsilon}\right)\left(x_{\epsilon}{ }^{2}-x_{\epsilon}\right)\right]}{\bar{x}^{2}\left(1-x_{\epsilon}\right)^{2}} .
\end{gathered}
$$

Now let us compute $\frac{\partial x_{t, h}}{\partial r_{t-k}}\left(M_{\epsilon}\right)$. The derivative of the multi-horizon demand 
function $(h>1)$ with respect to the contemporaneous return reads:

$$
\begin{gathered}
\frac{\partial x_{t, h}}{\partial r_{t}}\left(M_{\epsilon}\right)=\frac{1}{h} \frac{\partial}{\partial r_{t}}\left[\bar{x}+\frac{\bar{x}\left(1+r_{t}\right)}{1+\bar{x}\left(r_{t}+e_{t}\right)}+\frac{g(\bar{x})\left(1+r_{t}\right)}{1+g(\bar{x})\left(r_{t}+e_{t}\right)}+\right. \\
\left.\frac{g^{2}(\bar{x})\left(1+r_{t}\right)}{1+g^{2}(\bar{x})\left(r_{t}+e_{t}\right)}+\ldots+\frac{g^{h-2}(\bar{x})\left(1+r_{t}\right)}{1+g^{h-2}(\bar{x})\left(r_{t}+e_{t}\right)}\right]= \\
\frac{1}{h} \frac{\partial}{\partial r_{t}}\left[\bar{x}+(h-1) \frac{\bar{x}\left(1+r_{t}\right)}{1+\bar{x}\left(r_{t}+e_{t}\right)}\right]= \\
\frac{h-1}{h} \frac{\bar{x}-\bar{x}^{2}+\bar{x}^{2} e_{t}}{\left[1+\bar{x}\left(\bar{r}+e_{t}\right)\right]^{2}} .
\end{gathered}
$$

The derivative with respect to the lagged return is 0 if the lag $k$ is larger or equal to $h-1$. To compute it for the case $0<k<h-1$ let us denote:

$$
\tilde{x}=\frac{\bar{x}\left(1+r_{t-k}\right)}{1+\bar{x}\left(r_{t-k}+\bar{e}\right)}=\frac{g^{m}(\bar{x})\left(1+r_{t-k}\right)}{1+g^{m}(\bar{x})\left(r_{t-k}+\bar{e}\right)}, \forall m>0
$$

and

$$
g_{\epsilon}(x)=\frac{x(1+\bar{r})}{1+x\left(\bar{r}+e_{t}\right)} .
$$

Then using the property that $g\left(\tilde{x}\left(M_{\epsilon}\right)\right)=g(\tilde{x}(\bar{x}))=\bar{x}$, we obtain:

$$
\begin{gathered}
\frac{\partial x_{t, h}}{\partial r_{t-k}}\left(M_{\epsilon}\right)=\frac{1}{h} \frac{\partial}{\partial r_{t-k}}\left[\bar{x}+g_{\epsilon}(\bar{x})+g_{\epsilon}(g(\bar{x}))+g_{\epsilon}\left(g^{2}(\bar{x})\right)+\ldots+\right. \\
\left.g_{\epsilon}\left(g^{k-1}(\bar{x})\right)+(h-k-1) g_{\epsilon}\left(g^{k-1}(\tilde{x})\right)\right]= \\
\frac{h-k-1}{h} \frac{\partial}{\partial x} g_{\epsilon}\left(M_{\epsilon}\right) \frac{\partial}{\partial x} g^{k-1}\left(M_{\epsilon}\right) \frac{\partial}{\partial r_{t-k}} \tilde{x}= \\
\frac{h-k-1}{h} g_{\epsilon}^{\prime}(\bar{x})\left[g^{\prime}(\bar{x})\right]^{k-1} \frac{\partial}{\partial r_{t-k}} \tilde{x} .
\end{gathered}
$$

Computing derivatives in (A-14) yields:

$$
\begin{gathered}
\frac{\partial}{\partial x} g\left(M_{\epsilon}\right)=\frac{(1+\bar{r})[1+\bar{x}(\bar{r}+\bar{e})]-(\bar{r}+\bar{e})(1+\bar{r}) \bar{x}}{[1+\bar{x}(\bar{r}+\bar{e})]^{2}}= \\
\frac{1+\bar{r}}{[1+\bar{x}(\bar{r}+\bar{e})]^{2}}, \\
\frac{\partial}{\partial x} g_{\epsilon}\left(M_{\epsilon}\right)=\frac{1+\bar{r}}{\left[1+\bar{x}\left(\bar{r}+e_{t}\right)\right]^{2}}
\end{gathered}
$$

and

$$
\begin{gathered}
\frac{\partial}{\partial r_{t-k}} \tilde{x}\left(M_{\epsilon}\right)=\frac{\bar{x}[1+\bar{x}(\bar{r}+\bar{e})]-\bar{x}^{2}(1+\bar{r})}{[1+\bar{x}(\bar{r}+\bar{e})]^{2}}= \\
\frac{\bar{x}-\bar{x}^{2}+\bar{x}^{2} \bar{e}}{[1+\bar{x}(\bar{r}+\bar{e})]^{2}} .
\end{gathered}
$$

So finally for $k=1, \ldots, h-2$ we have:

$$
\frac{\partial x_{t, h}}{\partial r_{t-k}}\left(M_{\epsilon}\right)=\frac{h-k-1}{h} \frac{1+\bar{r}}{\left[1+\bar{x}\left(\bar{r}+e_{t}\right)\right]^{2}}\left[\frac{1+\bar{r}}{[1+\bar{x}(\bar{r}+\bar{e})]^{2}}\right]^{k-1} \frac{\bar{x}-\bar{x}^{2}+\bar{x}^{2} \bar{e}}{[1+\bar{x}(\bar{r}+\bar{e})]^{2}} .
$$


Then, using the fact that:

$$
\frac{\partial}{\partial e_{t-k}} \tilde{x}\left(M_{\epsilon}\right)=-\frac{\bar{x}^{2}(1+\bar{r})}{[1+\bar{x}(\bar{r}+\bar{e})]^{2}},
$$

we obtain:

$$
\frac{\partial x_{t, h}}{\partial e_{t-k}}\left(M_{\epsilon}\right)=-\frac{h-k-1}{h} \frac{1+\bar{r}}{\left[1+\bar{x}\left(\bar{r}+e_{t}\right)\right]^{2}}\left[\frac{1+\bar{r}}{[1+\bar{x}(\bar{r}+\bar{e})]^{2}}\right]^{k-1} \frac{\bar{x}^{2}(1+\bar{r})}{[1+\bar{x}(\bar{r}+\bar{e})]^{2}} .
$$

Using previous results, it is easy to notice that for $k=1, \ldots, h-1$ :

$$
\begin{aligned}
& \frac{\partial x_{t-1, h}}{\partial r_{t-k}}\left(M_{\epsilon}\right)=\frac{h-k}{h}\left[\frac{1+\bar{r}}{[1+\bar{x}(\bar{r}+\bar{e})]^{2}}\right]^{k-1} \frac{\bar{x}-\bar{x}^{2}+\bar{x}^{2} \bar{e}}{[1+\bar{x}(\bar{r}+\bar{e})]^{2}}, \\
& \frac{\partial x_{t-1, h}}{\partial e_{t-k}}\left(M_{\epsilon}\right)=-\frac{h-k}{h}\left[\frac{1+\bar{r}}{[1+\bar{x}(\bar{r}+\bar{e})]^{2}}\right]^{k-1} \frac{\bar{x}^{2}(1+\bar{r})}{[1+\bar{x}(\bar{r}+\bar{e})]^{2}} .
\end{aligned}
$$

Expressions (A-13),(A-15) - (A-17) can be simplified using the relation:

$$
\bar{x}=\frac{\bar{r}}{\bar{r}+\bar{e}}
$$

This gives:

$$
\begin{aligned}
\frac{\partial x_{t, h}}{\partial r_{t}}\left(M_{\epsilon}\right) & =\frac{h-1}{h} \frac{\bar{x}-\bar{x}^{2}+\bar{x}^{2} e_{t}}{\left[1+\bar{x}\left(\bar{r}+e_{t}\right)\right]^{2}}, \\
\frac{\partial x_{t, h}}{\partial r_{t-k}}\left(M_{\epsilon}\right) & =\frac{h-k-1}{h} \frac{\bar{x}-\bar{x}^{2}+\bar{x}^{2} \bar{e}}{(1+\bar{r})^{k}\left[1+\bar{x}\left(\bar{r}+e_{t}\right)\right]^{2}}, k=1, \ldots, h-2, \\
\frac{\partial x_{t-1, h}}{\partial r_{t-k}}\left(M_{\epsilon}\right) & =\frac{h-k}{h} \frac{\bar{x}(1-\bar{x})}{(1+\bar{r})^{k}}, k=1, \ldots, h-1, \\
\frac{\partial x_{t, h}}{\partial e_{t-k}}\left(M_{\epsilon}\right) & =-\frac{h-k-1}{h} \frac{1+\bar{r}}{\left[1+\bar{x}\left(\bar{r}+e_{t}\right)\right]^{2}} \frac{\bar{x}^{2}}{(1+\bar{r})^{k}}, k=1, \ldots, h-2, \\
\frac{\partial x_{t-1, h}}{\partial e_{t-k}}\left(M_{\epsilon}\right) & =-\frac{h-k}{h} \frac{\bar{x}^{2}}{(1+\bar{r})^{k}}, k=1, \ldots, h-1 .
\end{aligned}
$$

We can now replace expressions (A-18) in the equations for partial derivatives. Let us denote

$$
\begin{aligned}
a_{k} & =\sum_{h=k+2}^{H} \frac{h-k-1}{h} \xi_{0, h}, \\
b_{k} & =\sum_{h=k+1}^{H} \frac{h-k}{h} \xi_{0, h}, \\
c & =\sum_{h=1}^{H} \frac{h-1}{h} \xi_{0, h} .
\end{aligned}
$$

It is straightforward that $0<a_{k}<b_{k}<c<1$. In these terms: 


$$
\begin{gathered}
\frac{\partial F}{\partial r_{t-k}}\left(M_{\epsilon}\right)=\frac{a_{k} \frac{\bar{x}-\bar{x}^{2}+\bar{x}^{2} \bar{e}}{(1+\bar{r})^{k}\left[1+\bar{x}\left(\bar{r}+e_{t}\right)\right]^{2}}\left(\bar{x}+\bar{x}^{2} e_{t}-\bar{x}^{2}\right)+b_{k} \frac{\bar{x}(1-\bar{x})}{(1+\bar{r})^{k}}\left(x_{\epsilon}{ }^{2}-x_{\epsilon}\right)}{\bar{x}^{2}\left(1-x_{\epsilon}\right)^{2}}= \\
\frac{a_{k} \bar{x}^{2}(1-\bar{x}+\bar{x} \bar{e})\left(1-\bar{x}+\bar{x} e_{t}\right)-b_{k} \bar{x} x_{\epsilon}(1-\bar{x})\left(1-x_{\epsilon}\right)\left[1+\bar{x}\left(\bar{r}+e_{t}\right)\right]^{2}}{\bar{x}^{2}\left(1-x_{\epsilon}\right)^{2}(1+\bar{r})^{k}\left[1+\bar{x}\left(\bar{r}+e_{t}\right)\right]^{2}} \\
\frac{\partial F}{\partial r_{t}}\left(M_{\epsilon}\right)=\sum_{h=1}^{H} \xi_{0, h} \frac{h-1}{h} \frac{\bar{x}-\bar{x}^{2}+\bar{x}^{2} e_{t}}{\left[1+\bar{x}\left(\bar{r}+e_{t}\right)\right]^{2}} \frac{\left(\bar{x}+\bar{x}^{2} e_{t}-\bar{x}^{2}\right)}{\bar{x}^{2}\left(1-x_{\epsilon}\right)^{2}}-1= \\
\frac{c\left(1-\bar{x}+\bar{x} e_{t}\right)^{2}-\left(1-x_{\epsilon}\right)^{2}\left[1+\bar{x}\left(\bar{r}+e_{t}\right)\right]^{2}}{\left(1-x_{\epsilon}\right)^{2}\left[1+\bar{x}\left(\bar{r}+e_{t}\right)\right]^{2}} \\
\frac{\partial F}{\partial e_{t-k}}\left(M_{\epsilon}\right)= \\
\frac{-a_{k}(1+\bar{r}) \bar{x}^{2}\left(\bar{x}+\bar{x}^{2} e_{t}-\bar{x}^{2}\right)+b_{k} \bar{x}^{2} x_{\epsilon}\left(1-x_{\epsilon}\right)\left[1+\bar{x}\left(\bar{r}+e_{t}\right)\right]^{2}}{\bar{x}^{2}\left(1-x_{\epsilon}\right)^{2}(1+\bar{r})^{k}\left[1+\bar{x}\left(\bar{r}+e_{t}\right)\right]^{2}}
\end{gathered}
$$

We can now give explicit expressions for all the terms of equation (A-9):

$$
\begin{aligned}
& -\frac{F\left(M_{\epsilon}\right)}{\frac{\partial F}{\partial r_{t}}\left(M_{\epsilon}\right)}=-\left[\frac{x_{\epsilon}-\bar{x}+\bar{x} x_{\epsilon} e_{t}}{\bar{x}\left(1-x_{\epsilon}\right)}-\bar{r}\right] \times \\
& \frac{\left(1-x_{\epsilon}\right)^{2}\left[1+\bar{x}\left(\bar{r}+e_{t}\right)\right]^{2}}{c\left(1-\bar{x}+\bar{x} e_{t}\right)^{2}-\left(1-x_{\epsilon}\right)^{2}\left[1+\bar{x}\left(\bar{r}+e_{t}\right)\right]^{2}}, \\
& -\frac{\frac{\partial F}{\partial r_{t-k}}\left(M_{\epsilon}\right)}{\frac{\partial F}{\partial r_{t}}\left(M_{\epsilon}\right)}= \\
& -\frac{a_{k} \bar{x}^{2}(1-\bar{x}+\bar{x} \bar{e})\left(1-\bar{x}+\bar{x} e_{t}\right)-b_{k} \bar{x} x_{\epsilon}(1-\bar{x})\left(1-x_{\epsilon}\right)\left[1+\bar{x}\left(\bar{r}+e_{t}\right)\right]^{2}}{\bar{x}^{2}\left(1-x_{\epsilon}\right)^{2}(1+\bar{r})^{k}\left[1+\bar{x}\left(\bar{r}+e_{t}\right)\right]^{2}} \times \\
& \frac{\left(1-x_{\epsilon}\right)^{2}\left[1+\bar{x}\left(\bar{r}+e_{t}\right)\right]^{2}}{c\left(1-\bar{x}+\bar{x} e_{t}\right)^{2}-\left(1-x_{\epsilon}\right)^{2}\left[1+\bar{x}\left(\bar{r}+e_{t}\right)\right]^{2}}= \\
& -\frac{a_{k} \bar{x}(1-\bar{x}+\bar{x} \bar{e})\left(1-\bar{x}+\bar{x} e_{t}\right)-b_{k} x_{\epsilon}(1-\bar{x})\left(1-x_{\epsilon}\right)\left[1+\bar{x}\left(\bar{r}+e_{t}\right)\right]^{2}}{\bar{x}(1+\bar{r})^{k}\left[c\left(1-\bar{x}+\bar{x} e_{t}\right)^{2}-\left(1-x_{\epsilon}\right)^{2}\left(1+\bar{x}\left(\bar{r}+e_{t}\right)\right)^{2}\right]}, \\
& -\frac{\frac{\partial F}{\partial e_{t-k}}\left(M_{\epsilon}\right)}{\frac{\partial F}{\partial r_{t}}\left(M_{\epsilon}\right)}= \\
& -\frac{-a_{k}(1+\bar{r}) \bar{x}^{2}\left(\bar{x}+\bar{x}^{2} e_{t}-\bar{x}^{2}\right)+b_{k} \bar{x}^{2} x_{\epsilon}\left(1-x_{\epsilon}\right)\left[1+\bar{x}\left(\bar{r}+e_{t}\right)\right]^{2}}{\bar{x}^{2}\left(1-x_{\epsilon}\right)^{2}(1+\bar{r})^{k}\left[1+\bar{x}\left(\bar{r}+e_{t}\right)\right]^{2}} \times \\
& \frac{\left(1-x_{\epsilon}\right)^{2}\left[1+\bar{x}\left(\bar{r}+e_{t}\right)\right]^{2}}{c\left(1-\bar{x}+\bar{x} e_{t}\right)^{2}-\left(1-x_{\epsilon}\right)^{2}\left[1+\bar{x}\left(\bar{r}+e_{t}\right)\right]^{2}}= \\
& \frac{a_{k}(1+\bar{r})\left(\bar{x}+\bar{x}^{2} e_{t}-\bar{x}^{2}\right)-b_{k} x_{\epsilon}\left(1-x_{\epsilon}\right)\left[1+\bar{x}\left(\bar{r}+e_{t}\right)\right]^{2}}{(1+\bar{r})^{k}\left[c\left(1-\bar{x}+\bar{x} e_{t}\right)^{2}-\left(1-x_{\epsilon}\right)^{2}\left(1+\bar{x}\left(\bar{r}+e_{t}\right)\right)^{2}\right]} \text {. }
\end{aligned}
$$


Now let us linearize the terms of (A-9) with respect to the disturbance term $e_{t}$. We will need to use the relation:

$$
\frac{\partial x_{\epsilon}}{\partial e_{t}}=-\sum_{h=1}^{H} \xi_{0, h} \frac{h-1}{h} \frac{\bar{x}^{2}(1+\bar{r})}{(1+\bar{x}(\bar{r}+\bar{e}))^{2}}=-\frac{c \bar{x}^{2}}{1+\bar{r}} .
$$

The first term of (A-9) corresponds to the part of volatility that is independent of past realizations of the returns and dividend yields:

$$
-\frac{F\left(M_{\epsilon}\right)}{\frac{\partial F}{\partial r_{t}}\left(M_{\epsilon}\right)} \approx \bar{V} \widetilde{e}_{t}
$$

with

$$
\begin{gathered}
\bar{V}=\frac{c \bar{x}^{2}(1-\bar{e})-c \bar{x}+\bar{x}(1-\bar{x})(1+\bar{r})}{(1-c)(1+\bar{r})(1-\bar{x})^{2}}= \\
\frac{\bar{x}(1-\bar{x})(1+b r)(1-c)}{(1-c)(1+\bar{r})(1-\bar{x})^{2}}=\frac{\bar{x}}{1-\bar{x}}
\end{gathered}
$$

The second term gives both the autoregression coefficients in the equation for returns and the dependency of the volatility on past returns:

$$
-\frac{\frac{\partial F}{\partial r_{t-k}}\left(M_{\epsilon}\right)}{\frac{\partial F}{\partial r_{t}}\left(M_{\epsilon}\right)} \approx A_{k}+B_{k} \widetilde{e}_{t}
$$

with

$$
\begin{aligned}
A_{k} & =\frac{a_{k}-b_{k}}{(1-c)(1+\bar{r})^{k}}, \\
B_{k} & =\frac{\bar{x}(1-2 \bar{x})\left(c b_{k}-a_{k}\right)}{(1-\bar{x})(1+\bar{r})^{k+1}(1-c)} .
\end{aligned}
$$

In the same way we find:

$$
-\frac{\frac{\partial F}{\partial e_{t-k}}\left(M_{\epsilon}\right)}{\frac{\partial F}{\partial r_{t}}\left(M_{\epsilon}\right)} \approx C_{k}+D_{k} \widetilde{e}_{t}
$$

with

$$
\begin{aligned}
C_{k} & =-\frac{\bar{x}}{1-\bar{x}} A_{k}, \\
D_{k} & =-\frac{\bar{x}}{1-\bar{x}} B_{k} .
\end{aligned}
$$

The equation for returns thus reads:

$$
\widetilde{r}_{t}=\sum_{k=1}^{H-1}\left(A_{k}+B_{k} \widetilde{e}_{t}\right) \widetilde{r}_{t-k}-\bar{V} \sum_{k=1}^{H-1}\left(A_{k}+B_{k} \widetilde{e}_{t}\right) \widetilde{e}_{t-k}+\bar{V} \widetilde{e}_{t},
$$

which can also be written as:

$$
\widetilde{r}_{t}-\bar{V} \widetilde{e}_{t}=\sum_{k=1}^{H-1} A_{k}\left(\widetilde{r}_{t-k}-\bar{V} \widetilde{e}_{t-k}\right)+\sum_{k=1}^{H-1} B_{k}\left(\widetilde{r}_{t-k}-\bar{V} \widetilde{e}_{t-k}\right) \widetilde{e}_{t}
$$


Introducing the notation:

$$
\hat{r}_{t}=\widetilde{r}_{t}-\bar{V} \widetilde{e}_{t},
$$

we re-write the dynamics in terms of the error correction with respect to the one-scale rational equilibrium path, for which $\hat{r}_{t}=0$, as follows from theorem 3.2. In these terms we obtain:

$$
\hat{r}_{t}=\sum_{k=1}^{H-1} A_{k} \hat{r}_{t-k}+\left(\sum_{k=1}^{H-1} B_{k} \hat{r}_{t-k}\right) \widetilde{e}_{t},
$$

which closes the proof of the theorem. 